\title{
Equilibrium and matching under price controls
}

Citation for published version (APA):

Herings, P. J. J. (2015). Equilibrium and matching under price controls. Maastricht University, Graduate School of Business and Economics. GSBE Research Memoranda No. 001 https://doi.org/10.26481/umagsb.2015001

Document status and date:

Published: 01/01/2015

DOI:

10.26481/umagsb.2015001

Document Version:

Publisher's PDF, also known as Version of record

\section{Please check the document version of this publication:}

- A submitted manuscript is the version of the article upon submission and before peer-review. There can be important differences between the submitted version and the official published version of record.

People interested in the research are advised to contact the author for the final version of the publication, or visit the DOI to the publisher's website.

- The final author version and the galley proof are versions of the publication after peer review.

- The final published version features the final layout of the paper including the volume, issue and page numbers.

Link to publication

\footnotetext{
General rights rights.

- You may freely distribute the URL identifying the publication in the public portal. please follow below link for the End User Agreement:

www.umlib.nl/taverne-license

Take down policy

If you believe that this document breaches copyright please contact us at:

repository@maastrichtuniversity.nl

providing details and we will investigate your claim.
}

Copyright and moral rights for the publications made accessible in the public portal are retained by the authors and/or other copyright owners and it is a condition of accessing publications that users recognise and abide by the legal requirements associated with these

- Users may download and print one copy of any publication from the public portal for the purpose of private study or research.

- You may not further distribute the material or use it for any profit-making activity or commercial gain

If the publication is distributed under the terms of Article $25 \mathrm{fa}$ of the Dutch Copyright Act, indicated by the "Taverne" license above, 


\section{Maastricht University}

P. Jean-Jacques Herings

Equilibrium and Matching under Price Controls

RM/15/001

\section{GSBE}

Maastricht University School of Business and Economics

Graduate School of Business and Economics

P.O Box 616

NL- 6200 MD Maastricht

The Netherlands 


\title{
Equilibrium and Matching under Price Controls*
}

\author{
P. Jean-Jacques Herings ${ }^{\dagger}$ \\ January 13, 2015
}

\begin{abstract}
The paper considers a one-to-one matching with contracts model in the presence of price controls. This set-up contains two important streams in the matching literature, those with and those without monetary transfers, as special cases and allows for intermediate cases with some restrictions on the monetary transfers that are feasible. An adjustment process that ends with a stable outcome is presented, thereby proving the existence of stable outcomes. The process contains the deferred acceptance algorithm of Gale and Shapley (1962) and the approximate auction mechanism of Demange, Gale, and Sotomayor (1986) as special cases. The paper presents a notion of competitive equilibrium, called Drèze equilibrium, for this class of models, an extension of the concept as developed by Drèze (1975) for economies with divisible commodities subject to price controls. It is shown that Drèze equilibrium allocations are equivalent to allocations induced by stable outcomes. One implication is the existence of Drèze equilibria. Another implication is the equivalence of a competitive equilibrium concept and the concept of stable outcomes that is valid with and without monetary transfers as well as when monetary transfers are limited.
\end{abstract}

Keywords: Price Controls, Matching, Stable Outcomes, Competitive Equilibrium, Drèze Equilibrium.

JEL CODES: C71, C78, D45, D51.

*I would like to thank Tommy Andersson, Francis Bloch, Gabrielle Demange, Michael Greinecker, Dolf Talman, Fernando Vega-Redondo, Markus Walzl, and Alexander Westkamp for their very helpful comments on this paper.

${ }^{\dagger}$ P.J.J. Herings, Department of Economics, Maastricht University, P.O. Box 616, 6200 MD, Maastricht, The Netherlands. P.Herings@maastrichtuniversity.nl 


\section{Introduction}

Although standard competitive analysis assumes prices to be completely flexible, price controls and price regulations are actually very common. Nguyen and Whalley (1990), p. 667, write "Price controls have been employed by governments all over the world, during war and peace, in response to all manners of threats (both real and imaginary), and in all ages" and Levy (1991), p. 157, observes that "Price controls are pervasive in developing countries." Some typical examples include minimum wages in the labor market, minimum prices for agricultural products, price controls to reduce inflation (Cox, 1980), and upper bounds on rents. Andersson and Svensson (2014) report that as of 2011, legislated rent control existed in approximately 40 countries around the world and legislated minimum wages in around 200 countries.

The Arrow-Debreu theory of general equilibrium is a powerful tool to analyze resource allocation in market economies. However, many of the examples of markets with price controls, like the housing market or the labor market, are characterized by extensive heterogeneity. The standard competitive requirement that the goods traded in each market are homogeneous therefore leads to a very large number of extremely thin markets, which is at odds with the standard assumption of price-taking behavior. Moreover, in the presence of price controls, standard competitive equilibria often fail to exist as it can easily happen that every competitive equilibrium violates the restrictions as imposed by the price controls. The matching approach is therefore often believed to be more suitable for analyzing the discrete character as present in many markets, see for instance Hatfield, Plott, and Tanaka (2012b) and Hatfield, Kominers, Nichifor, Ostrovsky, and Westkamp (2013).

This paper starts from a one-to-one matching with contracts model in the spirit of Hatfield and Milgrom (2005). Buyers and sellers trade commodities by signing contracts, where a contract specifies a buyer, a seller, a commodity, and a price. We add the possibility that contracts are subject to price controls, which means that the price of a contract is subject to a price floor and a price ceiling, and therefore restricted to belong to some interval. The price floor is allowed to be equal to minus infinity and the price ceiling may be equal to plus infinity. Two important streams in the matching literature, those with and those without monetary transfers, can be seen as special cases. The case without monetary transfers as for instance studied in the marriage problem of Gale and Shapley (1962) follows by specifying both the lower and the upper bound on the price to be equal to zero. The case with unrestricted monetary transfers, the assignment model of Shapley and Shubik (1971) being a typical example, follows by setting the interval of feasible prices equal to the set of real numbers. Also intermediate cases, where price controls limit the monetary transfers that are feasible in non-degenerate ways, follow as special cases. Examples are Talman and Yang (2008) and Andersson and Svensson (2014), who consider the assignment 
model with price controls, and Hatfield, Plott, and Tanaka (2012b), who study how the presence of price controls leads to quality competition.

We present an adjustment process in the style of Kelso and Crawford (1982) that is shown to always terminate with a stable outcome in the discretized version of the model. The existence of a stable outcome in the model without a smallest monetary unit then follows from a simple limit argument. The adjustment process starts with a set of permitted contracts that is equal to all possible contracts and a set of tentatively accepted contracts that is equal to the empty set. In each step of the process, every unassigned buyer chooses one best element within the current set of permitted contracts. Next every seller tentatively accepts one best element within the set of choices by the unassigned buyers and the previous set of tentatively accepted contracts, leading to a new set of tentatively accepted contracts and assigned buyers. The process stops when none of the sellers reject any contract. Otherwise, the rejected contracts are removed from the set of permitted contracts and the process continues with the next iteration.

When applied to the marriage problem, the adjustment process is identical to the deferred acceptance algorithm of Gale and Shapley (1962). When applied to the assignment model, the adjustment process is identical to the generalization of the approximate auction mechanism with personalized prices as discussed in Section V of Demange, Gale, and Sotomayor (1986). When there are no ties, the adjustment process coincides with the generalized Gale-Shapley algorithm of Hatfield and Milgrom (2005).

We present a concept of competitive equilibrium in the matching with contracts model under price controls that does not suffer from lack of equilibrium existence. It is based on the contribution by Drèze (1975), who introduces price rigidities in the standard general equilibrium framework with divisible commodities, and presents an equilibrium concept where markets with binding price rigidities are cleared by quantity adjustments. We extend the equilibrium concept of Drèze (1975) to the matching with contracts framework and refer to the resulting equilibrium concept as Drèze equilibrium. Earlier contributions that have proposed equilibrium concepts under price controls for discrete set-ups are Talman and Yang (2008) and Andersson and Svensson (2014) under the names of constrained Walrasian equilibrium and rationing price equilibrium.

A Drèze equilibrium specifies commodity prices, a set of rationing constraints, and a feasible allocation. Prices should satisfy the lower bounds as expressed by price floors and the upper bounds as specified by price ceilings. Buyers and sellers determine their demand and supply for commodities by maximizing utility, thereby taking commodity prices and rationing constraints as given. Rationing constraints on the supply side can only occur in markets where the price floor is binding and serve as quantity adjustments to clear such markets. Similarly, rationing constraints on the demand side can only occur in markets 
with binding price ceilings. Markets are transparent, meaning that in the market of a given commodity it is not allowed that buyers and sellers are rationed simultaneously. In equilibrium, only the long side of the market is rationed and the amount of rationing is determined by the short side of the market. In the case of binding price floors, rationing constraints are determined by the demand side and are used to allocate the prevailing demand, if any, to all the sellers that are willing to supply the commodity. In the case of binding price ceilings, the supply side determines the amount of rationing of the buyers.

In competitive models, trade takes place anonymously via markets and it is not specified who trades with whom, contrary to the concept of a stable outcome as used in the matching approach. In this sense, a stable outcome provides more information than a concept of market equilibrium. On the other hand, a concept of market equilibrium specifies prices for all commodities, including the ones that are non-traded. It is therefore not immediate to compare stable outcomes as defined in matching theory with Drèze equilibria. We show that each stable outcome induces a unique allocation of commodities and that the same is true for Drèze equilibria. We can therefore compare allocations as resulting from the matching and the equilibrium approach, respectively. We demonstrate that both approaches lead to exactly the same predictions. The set of allocations corresponding to stable outcomes is identical to the set of Drèze equilibrium allocations. The existence of Drèze equilibria now follows as a corollary.

There are several papers that have established a connection between stable outcomes and competitive equilibria for the case of unlimited monetary transfers. Shapley and Shubik (1971) consider the assignment game and prove that the set of stable payoffs coincides with the set of competitive equilibrium payoffs. When allowing for personalized prices, such a result can be extended to many-to-many matching set-ups or even trading networks as has been demonstrated by Hatfield, Kominers, Nichifor, Ostrovsky, and Westkamp (2013). No parallel results exist for the case without monetary transfers. The current paper extends the Shapley and Shubik (1971) result in this direction and presents an equivalence result that holds both in the case of unlimited monetary transfers and in the absence of monetary transfers, as well as all for the intermediate cases where transfers are limited to some extent.

In the class of models under consideration, the set of stable outcomes coincides with the set of core outcomes. The relation between the core and competitive equilibrium is a classical problem in economics, dating back to Edgeworth (1881). In general, competitive equilibria belong to the core, but the core contains other allocations as well. In models of perfectly competitive economies, where agents are infinitely small, the core coincides with the set of competitive allocations. Debreu and Scarf (1963) obtain a core convergence result by showing that the set of core allocations shrinks to the competitive allocation when an economy is replicated. Aumann (1964) obtains a core equivalence result, showing 
that the core coincides with the set of competitive equilibrium allocations when there is a continuum of traders. This paper therefore delimits another class of economies where the core coincides with the set of equilibrium allocations. Moreover, agents need not be infinitely small. Where usually the equilibrium concepts are thought as more appealing in a set-up with many individuals that view themselves as an insignificant part of a large market and the cooperative concepts are thought to be more appropriate in situations with few players, both approaches coincide here irrespective of the number of agents.

The paper is organized as follows. Section 2 presents the model of matching with contracts under price controls and shows how many contributions in the literature are included as special cases. Section 3 presents the notion of stable outcome. To illustrate the model and the stability concept, the quality competition model of Hatfield, Plott, and Tanaka (2012b) is examined in detail. Section 4 shows stable outcomes to exist by an adjustment process that generalizes both the deferred acceptance algorithm of Gale and Shapley (1962) and the approximate auction mechanism of Demange, Gale, and Sotomayor (1986). Section 5 extends the seminal work by Drèze (1975) to the discrete matching framework and presents the notion of Drèze equilibrium. The equilibrium concept is illustrated for the quality competition model. Section 6 is devoted to the equivalence between allocations induced by stable outcomes and Drèze equilibrium allocations. Section 7 concludes.

\section{Matching under Price Controls}

There is a finite set of buyers $B$ and a finite set of sellers $S$ who trade commodities in a finite set $L$ and a good labeled 0 , which we call money. The set of all agents is $I=B \cup S$. Trade in commodities takes place by signing contracts, where a contract is an element $y=(b, s, \ell, m) \in B \times S \times L \times \mathbb{R}$. A given contract $y=(b, s, \ell, m)$ involves a buyer $\beta(y)=b$, a seller $\sigma(y)=s$, a commodity $\lambda(y)=\ell$, and an amount of money $\mu(y)=m$. The set of agents involved in contract $y$ is $\iota(y)=\{\beta(y), \sigma(y)\}$. We consider a one-to-one matching set-up, so a buyer signs a contract with at most one seller and a seller with at most one buyer.

For a set of contracts $Y \subset B \times S \times L \times \mathbb{R}$, the set of contracts involving buyer $b$ is given by $Y^{b}=Y \cap \beta^{-1}(\{b\})=\{y \in Y \mid \beta(y)=b\}$ and the set of contracts involving seller $s$ by $Y^{s}=Y \cap \sigma^{-1}(\{s\})=\{y \in Y \mid \sigma(y)=s\}$. Similarly, the set of contracts involving commodity $\ell$ is given by $Y^{\ell}=Y \cap \lambda^{-1}(\{\ell\})=\{y \in Y \mid \lambda(y)=\ell\}$.

The amount of money involved in a contract may be subject to price controls. The price of a commodity $\ell \in L$ is subject to a price floor $\underline{p}_{\ell} \in\{-\infty\} \cup \mathbb{R}$ and a price ceiling $\bar{p}_{\ell} \in \mathbb{R} \cup\{+\infty\}$. The set of admissible prices is now equal to $P=\left\{p \in \mathbb{R}^{L} \mid \underline{p} \leq p \leq \bar{p}\right\}$. To ensure that the set of admissible prices is non-empty, it is assumed that $\underline{p} \leq \bar{p}$. One extreme 
but highly relevant case is where no monetary transfers are allowed. This is achieved by setting $\underline{p}_{\ell}=\bar{p}_{\ell}=0$. In applications related to social housing, it is common that the rent $m_{\ell}$ to be paid for an apartment of type $\ell$ is fixed, which corresponds to setting $\underline{p}_{\ell}=\bar{p}_{\ell}=m_{\ell}$. The case with unlimited monetary transfers follows by specifying $\underline{p}_{\ell}=-\infty$ and $\bar{p}_{\ell}=+\infty$. Both price floors and price ceilings are allowed to be negative.

In many settings, it is not possible for every buyer to supply every commodity to every seller. Some specific examples are presented later. The set of feasible trades is given by $T$, a subset of $B \times S \times L$. We assume that for every commodity its set of traders has a product structure. Whenever there is a trade in $T$ involving commodity $\ell$ by buyer $b^{1}$ and seller $s^{1}$ as well as a trade by buyer $b^{2}$ and seller $s^{2}$, then there is also a trade in $T$ involving commodity $\ell$ by buyer $b^{1}$ and seller $s^{2}$, and a trade in $T$ involving commodity $\ell$ by buyer $b^{2}$ and seller $s^{1}$. Equivalently, for every $\ell \in L$, the graph with as nodes the buyers $\beta\left(\bar{Y}^{\ell}\right)$ and the sellers $\sigma\left(\bar{Y}^{\ell}\right)$ and an edge between a buyer $b$ and a seller $s$ if and only if $(b, s, \ell) \in T$ is a complete bipartite graph. This assumption is without loss of generality, since any form of trading restrictions can be incorporated in the notion of a commodity. In the extreme, we could define as many commodities as there are trades.

The set $T$ generates the set of feasible contracts $\bar{Y}$ defined as the set of all contracts with the trade in $T$ and the amount of money satisfying the restrictions imposed by the price controls, so

$$
\bar{Y}=\left\{y \in B \times S \times L \times \mathbb{R} \mid(\beta(y), \sigma(y), \lambda(y)) \in T \text { and } \underline{p}_{\lambda(y)} \leq \mu(y) \leq \bar{p}_{\lambda(y)}\right\}
$$

The commodities that can be traded by an agent $i \in I$ are defined by

$$
L^{i}=\lambda\left(\bar{Y}^{i}\right)=\left\{\ell \in L \mid \exists y \in \bar{Y}^{i} \text { such that } \lambda(y)=\ell\right\} .
$$

It holds that $\cup_{b \in B} L^{b}=\cup_{s \in S} L^{s}=L$.

For $\ell \in L$, let $e(\ell)$ be the $\ell$-th unit vector in $\mathbb{R}^{L}$. Then the set of feasible contracts $\bar{Y}$ defines the consumption set $X^{b}$ of a buyer $b \in B$ to be equal to

$$
X^{b}=\mathbb{R} \times\left(\left\{0^{L}\right\} \cup\left\{e(\ell) \mid \ell \in L^{b}\right\}\right),
$$

where $0^{L}$ denotes the zero vector in $\mathbb{R}^{L}$ and corresponds to the case where the buyer does not sign any contract. The unit vector $e(\ell)$ results when buyer $b$ signs a contract involving commodity $\ell$. The first component of a vector $x^{b} \in X^{b}$ denotes the amount of money consumed and is denoted by $x_{0}^{b}$. If buyer $b$ signs a contract involving an amount of money $m$, then $x_{0}^{b}=-m$. If buyer $b$ does not sign a contract, then $x_{0}^{b}=0$.

The consumption set $X^{s}$ of a seller $s$ is given by

$$
X^{s}=\mathbb{R} \times\left(\left\{0^{L}\right\} \cup\left\{-e(\ell) \mid \ell \in L^{s}\right\}\right),
$$


where we make the usual convention that the supply of commodity $\ell$ by a seller $s$ results in a negative consumption, $x_{\ell}^{s}=-e(\ell)$. If seller $s$ signs a contract involving an amount of money $m$, then $x_{0}^{s}=m$. If seller $s$ does not sign a contract, then $x_{0}^{s}=0$.

We denote $X=\prod_{i \in I} X^{i}$.

The preferences of an agent $i \in I$ are represented by a utility function $u^{i}: X^{i} \rightarrow \mathbb{R}$. The concept of a commodity encompasses all utility relevant information. For a buyer the utility of a commodity does not depend on the agent delivering it, and for a seller the utility of a commodity is independent of the identity of its buyer. This assumption is without loss of generality. Whenever such information is relevant, it should be included in the description of a commodity.

Utility functions are assumed to be continuous and strongly monotonic in $x_{0}$. There are limits to the monetary transfers buyers are willing to make for commodities without price ceilings. For every $b \in B$, for every $\ell \in L^{b}$ such that $\bar{p}_{\ell}=+\infty$, there is $m \in \mathbb{R}$ such that $u^{b}(-m, e(\ell)) \leq u^{b}\left(0,0^{L}\right)$. There are limits to the monetary transfers sellers are willing to make for commodities without price floors. For every $s \in S$, for every $\ell \in L^{s}$ such that $\underline{p}_{\ell}=-\infty$, there is $m \in \mathbb{R}$ such that $u^{s}(m,-e(\ell)) \leq u^{s}\left(0,0^{L}\right)$. Commodities are allowed to be bads, so for a buyer it might be that for some commodity $\ell$ it holds that $u^{b}(0, e(\ell))<u^{b}\left(0,0^{L}\right)$, and for a seller that $u^{s}(0,-e(\ell))>u^{s}\left(0,0^{L}\right)$. Quasi-linear utility functions are a prominent and commonly used example of utility functions satisfying our assumptions.

The primitives of an economy are summarized by $\mathcal{E}=\left(T, \underline{p}, \bar{p},\left(u^{i}\right)_{i \in I}\right)$. Although simple, this model includes a number of important special cases.

EXAmple 2.1: Gale and Shapley (1962), marriage problem.

A community consists of $n$ men and $n$ women. Each person ranks those of the oppositive sex in accordance with his or her preferences for a marriage partner. Monetary transfers are not allowed. We define $B$ as the set of men, $S$ as the set of women, and the set of commodities as $L=B \times S$. This is clearly a case with many trading restrictions, since buyers and sellers are both restricted to trade in the commodity carrying their label, $T=\{(b, s, \ell) \in B \times S \times L \mid \ell=(b, s)\}$. A commodity corresponds to a contract between a man and a woman. The absence of monetary transfers is achieved by setting $\underline{p}=\bar{p}=0^{L}$. Utility functions are now specified in accordance with each person's ranking of the partners of opposite sex, with the utility of remaining single strictly below the lowest utility assigned to a partner when $x_{0}=0$. Since monetary transfers are not possible, the specification of the utility function is immaterial for other values of $x_{0}$. Since there are as many commodities as there are trades, $T$ trivially has a product structure. 
ExAmple 2.2: Shapley and Shubik (1971), assignment model.

There are $m$ homeowners in the real estate market, and $n$ prospective purchasers. Homeowner $s$ values his house at $c_{s}$ dollars, while purchaser $b$ values the same house at $h_{b s}$ dollars. There are no restrictions on monetary transfers. We define $B$ as the set of prospective purchasers, $S$ as the set of homeowners, and the set of commodities as $L=S$. A commodity corresponds to a house. Again, there are many trading restrictions, since a seller is restricted to trade in the house baring his label, $T=\{(b, s, \ell) \in B \times S \times L \mid \ell=s\}$. There are no restrictions on monetary transfers, so for every $\ell \in L$ we set $\underline{p}_{\ell}=-\infty$ and $\bar{p}_{\ell}=+\infty$. The utility of a buyer $b \in B$ is given by

$$
u^{b}(x)=x_{0}+\sum_{s \in S} h_{b s} x_{s}
$$

The utility of a seller $s \in S$ is equal to

$$
u^{s}(x)=x_{0}+c_{s} x_{s}
$$

It is easily verified that this application satisfies all the assumptions made before.

ExAmple 2.3: Talman and Yang (2008), assignment model with price controls and quasi-linear utility functions.

The model is the one of Example 2.2 with $c_{s}=0$ for every $s \in S$. Moreover, price controls restrict monetary transfers. Price controls are expressed by price floors $p \in \mathbb{N}_{0}^{L}$ and price ceilings $\bar{p} \in \mathbb{N}_{0}^{L}$, where $\mathbb{N}_{0}$ denotes the set of natural numbers including 0 .

ExAmple 2.4: Andersson and Svensson (2014), assignment model with price controls and general utility functions.

The model is the one of Example 2.2, except that utility functions need not be quasi-linear but are only assumed to be continuous and strongly monotonic in $x_{0}$. Moreover, there are price controls expressed by price floors $\underline{p} \in \mathbb{R}^{L}$ and price ceilings $\bar{p} \in(\mathbb{R} \cup\{+\infty\})^{L}$. The price floors correspond to the sellers' reservation utilities, so $u^{s}(\underline{p},-e(s))=u^{s}\left(0,0^{L}\right)$, $s \in S$.

ExAmple 2.5: Hatfield, Plott, and Tanaka (2012b), quality competition in the presence of price controls.

Several authors have argued that the presence of price controls affects the commodity which is traded on the market. For instance, Leffler (1982) argues that price ceilings give rise to the supply of inefficiently low quality. Other authors, like Feldstein (1973), have studied the effect of minimum wages on job quality, and Agell and Lommerud (1997) analyze the effects of minimum wages on worker's skill formation. To study the effects of price 
controls on traded quality, Hatfield, Plott, and Tanaka (2012b) develop a model of quality competition.

The set of commodities is $L=\{1, \ldots, \bar{\ell}\}$. Commodities have universally agreed upon qualities, with the quality of commodity $\ell$ exceeding the quality of commodity $k$ if and only if $\ell \geq k$. Commodities are therefore referred to as qualities. There is a price control that specifies a common price floor $f$ for all qualities: for every $\ell \in L, p_{\ell}=f$. There are no price ceilings, so for every $\ell \in L, \bar{p}_{\ell}=+\infty$. The value for buyer $b$ of procuring any quality is given by $v^{b} \geq 0$, and $v_{\ell}$ denotes the additional utility derived from procuring quality $\ell$. To obtain a unique utility representation, it is assumed that $v_{1}=0$. For $\ell=2, \ldots, \bar{\ell}$, we define $\Delta v_{\ell}=v_{\ell}-v_{\ell-1}$ as the difference in utility between quality $\ell$ and quality $\ell-1$. It is assumed that $\Delta v_{\ell}$ is positive. The utility of a buyer $b \in B$ is then given by

$$
u^{b}(x)=x_{0}+v^{b} \sum_{\ell \in L} x_{\ell}+\sum_{\ell \in L} v_{\ell} x_{\ell}
$$

Sellers produce at most one quality. The cost for seller $s$ of producing any quality is given by $c^{s} \geq 0$, and $c_{\ell}$ denotes the additional cost of producing commodity $\ell$. To obtain a unique representation of the costs, it is assumed that $c_{1}=0$. For $\ell=2, \ldots, \bar{\ell}$, we define $\Delta c_{\ell}=c_{\ell}-c_{\ell-1}$ as the increase in costs to go from quality $\ell-1$ to quality $\ell$. It is assumed that $\Delta c_{\ell}$ is positive. The utility of a seller $s \in S$ is given by the profit

$$
u^{s}(x)=x_{0}+c^{s} \sum_{\ell \in L} x_{\ell}+\sum_{\ell \in L} c_{\ell} x_{\ell}
$$

The set of applications of the general model can be easily expanded. For instance, upper bounds on prices can be used to model buyers with liquidity constraints as in Maskin (2000) and restrictions on the set of feasible trades can be used to analyze trading networks as in Corominas-Bosch (2004).

\section{Stable outcomes}

In this section, we present the solution concept of stability, a concept that is suggested by both theoretical, empirical, and experimental work, see e.g. Roth (1991) and Hatfield, Plott, and Tanaka (2012a).

A set of contracts $A \subset \bar{Y}$ is an outcome if each agent is involved in at most one contract, so the cardinality of every set $A^{i}$ is at most one. The set of all outcomes is denoted by the collection $\mathcal{A}=\left\{A \subset \bar{Y}|\forall i \in I,| A^{i} \mid \leq 1\right\}$. In particular, it holds that $\emptyset \in \mathcal{A}$. For $i \in \mathcal{I}$, the collection $\mathcal{A}^{i}$ is defined as the collection containing the empty set together with all sets consisting of a single contract that involves agent $i$ and corresponds to the set of possible choices for agent $i$. More formally, $\mathcal{A}^{i}=\{A \in \mathcal{A} \mid \forall y \in A, i \in \iota(y)\}$. An 
outcome $A$ generates the unique allocation $\xi(A) \in X$ defined as follows. For every $b \in B$, if $A^{b}=\emptyset$, then $\xi^{b}(A)=\left(0,0^{L}\right)$; otherwise, let $y$ be the unique contract in $A^{b}$ and define $\xi^{b}(A)=(-\mu(y), e(\lambda(y)))$. For every $s \in S$, if $A^{s}=\emptyset$, then $\xi^{s}(A)=\left(0,0^{L}\right)$; otherwise, let $y$ be the unique contract in $A^{s}$ and define $\xi^{s}(A)=(\mu(y),-e(\lambda(y)))$. Since every contract specifies the delivery of a commodity from a seller to a buyer in exchange for a particular quantity of money, it holds that

$$
\sum_{i \in I} \xi^{i}(A)=0
$$

The utility of agent $i \in I$ derived from outcome $A \in \mathcal{A}$ is given by

$$
U^{i}(A)=u^{i}\left(\xi^{i}(A)\right)
$$

The choice relation $C^{i}$ of agent $i \in I$ is defined by

$$
C^{i}(Y)=\arg \max _{\left\{A^{i} \in \mathcal{A}^{i} \mid A^{i} \subset Y^{i}\right\}} U^{i}\left(A^{i}\right), \quad Y \subset \bar{Y} .
$$

The relation $C^{i}$ might be empty-valued. This could for instance occur when the set $Y$ is open. In the sequel, we will apply $C^{i}$ to finite sets $Y$, which clearly guarantees that $C^{i}$ is not empty-valued. The next definition rewrites the stability concept as defined in Roth and Sotomayor (1990) to our set-up.

Definition 3.1: An outcome $A \in \mathcal{A}$ is stable if:

1. For every $i \in I, A^{i} \in C^{i}(A)$.

2. For every $z=(b, s, \ell, m) \in \bar{Y}$ it holds that $U^{b}(\{z\}) \leq U^{b}(A)$ or $U^{s}(\{z\}) \leq U^{s}(A)$.

A stable outcome $A$ involves only contracts in the set of feasible contracts $\bar{Y}$ and at most one contract for each agent. The first condition in Definition 3.1 corresponds to individual rationality. Every agent $i$ should weakly prefer his current contract to staying inactive. The second condition requires absence of blocking by a two-agent coalition. It now follows easily that an outcome is stable if and only if it belongs to the core.

To illustrate the usefulness of the model and the richness of the stable outcomes that result, we now examine the model of Example 2.5 in a bit more detail. Suppose first that there is a single quality $\ell$ and there are no price controls, so $\bar{Y}=B \times S \times\{\ell\} \times \mathbb{R}$. In that case it is easily shown that an outcome $A$ is stable if and only if every contract in $A$ trades at the same price $p_{\ell}$ and $A$ is constrained efficient, i.e. maximizes the sum of utilities over outcomes in $\mathcal{A}$. For a given stable outcome $A$, we define

$$
\begin{aligned}
\underline{q}_{\ell}^{B} & =\sup _{b \in B \backslash \beta(A)} v^{b}+v_{\ell}, \\
\underline{q}_{\ell}^{S} & =\sup _{s \in \sigma(A)} c^{s}+c_{\ell}, \\
\underline{q}_{\ell} & =\max \left\{\underline{q}_{\ell}^{B}, \underline{q}_{\ell}^{S}\right\},
\end{aligned}
$$


and

$$
\begin{aligned}
\bar{q}_{\ell}^{B} & =\inf _{b \in \beta(A)} v^{b}+v_{\ell}, \\
\bar{q}_{\ell}^{S} & =\inf _{s \in S \backslash \sigma(A)} c^{s}+c_{\ell}, \\
\bar{q}_{\ell} & =\min \left\{\bar{q}_{\ell}^{B}, \bar{q}_{\ell}^{S}\right\} .
\end{aligned}
$$

The amount $\underline{q}_{\ell}^{B}$ corresponds to the highest willingness to pay of buyers who are not involved in any contract in $A$, and $\underline{q}_{\ell}^{S}$ to the highest cost of a seller who is involved in $A$. Although $\underline{q}_{\ell}^{B}$ and $\underline{q}_{\ell}^{S}$ may depend on $A$, it holds that $\underline{q}_{\ell}$ is independent of the choice for the stable outcome $A$. Similarly, $\bar{q}_{\ell}^{B}$ corresponds to the lowest willingness to pay of buyers who are involved in some contract in $A$, and $\bar{q}_{\ell}^{S}$ the lowest cost of a seller who is not involved in any contract in $A$. It holds that $\bar{q}_{\ell}$ is independent of the choice for the stable outcome $A$. The common price $p_{\ell}$ should be such that $\underline{q}_{\ell} \leq p_{\ell} \leq \bar{q}_{\ell}$. The price $p_{\ell}$ should be sufficiently high such that a buyer without a contract is not willing to buy and every seller with a contract is willing to supply. Similarly, the price $p_{\ell}$ should be sufficiently low such that a buyer with a contract is willing to buy and a seller without a contract is not willing to supply.

We now consider the case where a price floor $f$ is imposed. The set of contracts is then equal to $\bar{Y}=\{y \in B \times S \times L \times \mathbb{R} \mid \mu(y) \geq f\}$. The following result has been shown in Hatfield, Plott, and Tanaka (2012b). The economy $\mathcal{E}$ is said to be convex if $\Delta v_{\ell}$ is non-increasing in $\ell$ and $\Delta c_{\ell}$ is non-decreasing in $\ell$.

TheOREM 3.2: Consider a convex economy $\mathcal{E}$ with price floor $f$ and assume there is a unique quality $\ell^{*}<\bar{\ell}$ that maximizes the surplus $v_{\ell}-c_{\ell}$. A stable outcome $A$ exists. Moreover, we have the following cases.

1. $f<\underline{q}_{\ell^{*}}$. The outcome $A$ is efficient and there is $m \in\left[\underline{q}_{\ell^{*}}, \bar{q}_{\ell^{*}}\right]$ such that for every $y \in A$ it holds that $\mu(y)=m$.

2. $\bar{q}_{\ell}<f<\underline{q}_{\ell+1}-\Delta v_{\ell+1}$ for some $\ell \geq \ell^{*}$. For every $y \in A$ it holds that

$$
[\lambda(y)=\ell \text { and } \mu(y)=f] \text { or }\left[\lambda(y)=\ell+1 \text { and } \mu(y)=f+\Delta v_{\ell+1}\right] .
$$

Moreover, it holds that

$$
\beta(A) \in \arg \max _{\hat{B} \subset B} \sum_{b \in \hat{B}}\left(v^{b}+v_{\ell}-f\right) .
$$

3. $\bar{q}_{\ell+1}-\Delta v_{\ell+1}<f<\underline{q}_{\ell+1}$ for some $\ell \geq \ell^{*}$. The outcome $A$ is constrained efficient for the set of feasible contracts $B \times S \times\{\ell+1\} \times \mathbb{R}$ and there is $m \in\left[\underline{q}_{\ell+1}, \bar{q}_{\ell+1}\right]$ such that for every $y \in A$ it holds that $\mu(y)=m$. 


\begin{tabular}{|l|lll|}
\hline & 1 & 2 & 3 \\
\hline$b_{1}$ & 7 & 8 & 9 \\
$b_{2}$ & 6 & 7 & 8 \\
$b_{3}$ & 2 & 3 & 4 \\
\hline
\end{tabular}

\begin{tabular}{|l|lll|}
\hline & 1 & 2 & 3 \\
\hline$s_{1}$ & 1 & 3 & 6 \\
$s_{2}$ & 2 & 4 & 7 \\
$s_{3}$ & 4 & 6 & 9 \\
\hline
\end{tabular}

Table 1: Valuations and costs in an economy with $B=\left\{b_{1}, b_{2}, b_{3}\right\}, S=\left\{s_{1}, s_{2}, s_{3}\right\}$, and $L=\{1,2,3\}$.

The first case of Theorem 3.2 corresponds to a price floor below the lower bound on the common price in case only the efficient quality $\ell^{*}$ is available. In this case the price floor is ineffective and the set of stable outcomes coincides with the one in the absence of price floors.

It is tempting to replace the assumption in the first case by $f<\bar{q}_{\ell^{*}}$. Although any stable outcome in the absence of price floors remains stable as there are less blocking opportunities, new possibilities arise.

Consider, for instance, an economy with three buyers, three sellers, and three qualities, where valuations and costs are as depicted in Table 1 . It can easily be verified that $\ell^{*}=1$, $\underline{q}_{1}=2, \bar{q}_{1}=4, \underline{q}_{2}=4, \bar{q}_{2}=6, \underline{q}_{3}=7$, and $\bar{q}_{3}=8$. When the price floor $f$ is less than or equal to 4 , every efficient stable outcome $A$ involves two contracts, $\beta(A)=\left\{b_{1}, b_{2}\right\}$, $\sigma(A)=\left\{s_{1}, s_{2}\right\}$, only quality 1 is traded, $\lambda(A)=\{1\}$, and every contract in $A$ trades at a price $m$ in between $f$ and $\bar{q}_{1}=4$.

However, even when the price floor is less than or equal to $\bar{q}_{1}=4$, but is greater than or equal to $\underline{q}_{1}=2$, there are also constrained inefficient stable outcomes. Consider, for instance, the outcome $A=\left\{\left(b_{1}, s_{1}, 2, f+1\right),\left(b_{2}, s_{2}, 1, f\right)\right\}$, where buyer $b_{1}$ trades quality 2 with seller $s_{1}$ against the price $f+1$ and buyer $b_{2}$ trades quality 1 with seller $s_{2}$ against the price $f$. We verify next that $A$ is stable. Every contract in $A$ obeys individual rationality. Suppose there is a contract $z=(b, s, \ell, m)$ which gives both $b$ and $s$ strictly higher utility. It holds that $U^{b_{1}}(A)=7-f$, so to give $b_{1}$ strictly higher utility, it should hold that $\ell=1$ and $m<f$, or $\ell=2$ and $m<f+1$, or $\ell=3$ and $m<f+2$. The first case is not possible in the presence of a price floor equal to $f$. The latter two cases are not possible since the utility for $s$ would be lower than $U^{s}(A)$. It follows that $b \neq b^{1}$. The same argument can be used to show that $b \neq b^{2}$. Buyer $b_{3}$ cannot get a positive utility by consuming $\ell^{*}=1$ against price $f$, and no seller can produce quality 2 against a cost lower than $v^{b_{3}}+v_{2}=3$ or quality 3 against a cost lower than $v^{b_{3}}+v_{3}=4$, so $b \neq b_{3}$.

The second case in Theorem 3.2 involves trade in only one quality, either $\ell$ or $\ell+1$, or both qualities $\ell$ and $\ell+1$. The price of any contract where quality $\ell$ is traded is equal to $f$, the price of any contract with trade in quality $\ell+1$ is equal to $f+\Delta v_{\ell+1}$, which 
makes buyers indifferent between these two types of contract. Trade in quality $\ell+1$ corresponds to trade in an inefficiently high quality and the same holds for trade in quality $\ell$ whenever $\ell>\ell^{*}$. The outcome is not even constrained efficient for the set of feasible contracts $B \times S \times\{\ell, \ell+1\} \times \mathbb{R}$, since whenever there is trade in quality $\ell+1$ against a price $f+\Delta v_{\ell+1}$, the seller of such a contract would be strictly better off by trading quality $\ell$ against a price $f$, leaving the buyer of such a contract indifferent.

The condition $\bar{q}_{\ell}<f<\underline{q}_{\ell+1}-\Delta v_{\ell+1}$ for some $\ell \geq \ell^{*}$ in the second case of Theorem 3.2 might be empty. For instance, in the example of Table 1, it holds that $\bar{q}_{1}=4$, whereas $\underline{q}_{2}-\Delta v_{2}=4-1=3$. Similarly, it holds that $\bar{q}_{2}=6$, whereas $\underline{q}_{3}-\Delta v_{3}=7-1=6$. It is tempting to reduce the lower bound on $f$ to $\underline{q}_{\ell}$. However, we can use the same construction as before to show that when the price floor $f$ is in between $\underline{q}_{2}=4$ and 5 there are stable outcomes $A$ with $\lambda(A)=\{1,2\}$, so violating the assertion in Case 2 that for every $y \in A$ it holds that $\lambda(y) \in\{2,3\}$.

In the third case of Theorem 3.2 only quality $\ell+1$ is traded against a price in between $\underline{q}_{\ell+1}$ and $\bar{q}_{\ell+1}$. In this case, the traded quality is again inefficiently high. The outcome is constrained efficient when feasible contracts are restricted to quality $\ell+1$. In the example of Table 1 , the condition $\bar{q}_{\ell+1}-\Delta v_{\ell+1}<f<\underline{q}_{\ell+1}$ for some $\ell \geq \ell^{*}$ of Case 3 is empty as well, since $5=6-1=\bar{q}_{2}-\Delta v_{2}>\underline{q}_{2}=4$ and $7=8-1=\bar{q}_{3}-\Delta v_{3}=\underline{q}_{3}=7$.

\section{Existence of Stable Outcomes}

In this section, we present an adjustment process in the spirit of Kelso and Crawford (1982) that ends with a stable outcome.

Consider some $t=(b, s, \ell) \in T$. Without loss of generality, we assume in the formulation of the adjustment process that there is $m \in\left[\underline{p}_{\ell}, \bar{p}_{\ell}\right] \cap \mathbb{R}$ such that $u^{b}(-m, e(\ell)) \geq u^{b}\left(0,0^{L}\right)$ and $u^{s}(m,-e(\ell)) \geq u^{s}\left(0,0^{L}\right)$. Otherwise, a trade can never be part of a stable outcome by individual rationality and can be dropped from $T$.

We first reduce the set of possible contracts to a finite set. Consider some $t=(b, s, \ell) \in$ $T$. The amount of money specified in a contract involving trade $t$ is restricted to belong to an interval $\left[\underline{m}_{t}, \bar{m}_{t}\right]$, where $\underline{m}_{t}$ and $\bar{m}_{t}$ are both finite.

To define $\underline{m}_{t}$, we distinguish two cases. In the first case, $\underline{p}_{\ell}=-\infty$. Let $\underline{m}_{t} \in \mathbb{R}$ be the unique amount of money such that $u^{s}\left(\underline{m}_{t},-e(\ell)\right)=u^{s}\left(0,0^{L}\right)$. Since it has been assumed that for every $\ell \in L^{s}$ such that $\underline{p}_{\ell}=-\infty$, there is $m \in \mathbb{R}$ such that $u^{s}(m,-e(\ell)) \leq u^{s}\left(0,0^{L}\right)$ and there is $m \in\left[\underline{p}_{\ell}, \bar{p}_{\ell}\right] \cap \mathbb{R}$ such that $u^{s}(m,-e(\ell)) \geq u^{s}\left(0,0^{L}\right)$, and $u^{s}$ is strongly monotonic in $x_{0}$, it holds that $\underline{m}_{t}$ is well-defined, uniquely determined, and less than or equal to $\bar{p}_{\ell}$. Individual rationality of seller $s$ implies that $\underline{m}_{t}$ is the lowest amount of money that $s$ is ever going to accept for a contract involving trade $t$. In the second case, it holds that $\underline{p}_{\ell} \neq-\infty$. 
Either $u^{s}\left(\underline{p}_{\ell},-e(\ell)\right) \geq u^{s}\left(0,0^{L}\right)$ and we define $\underline{m}_{t}=\underline{p}_{\ell^{\prime}}$, or $u^{s}\left(\underline{p}_{\ell},-e(\ell)\right)<u^{s}\left(0,0^{L}\right)$ and we define $\underline{m}_{t} \in \mathbb{R}$ as the unique amount of money such that $u^{s}\left(\underline{m}_{t},-e(\ell)\right)=u^{s}\left(0,0^{L}\right)$. By the same argument as before, $\underline{m}_{t}$ is well-defined, uniquely determined, and less than or equal to $\bar{p}_{\ell}$. The lower bound on the price of commodity $\ell$, together with individual rationality of seller $s$, implies that $\underline{m}_{t}$ is the lowest amount of money that $s$ is ever going to accept for a contract involving trade $t$.

Next, we define $\bar{m}_{t}$ in a similar way. If $\bar{p}_{\ell}=+\infty$, then let $\bar{m}_{t} \in \mathbb{R}$ be the unique amount of money such that $u^{b}\left(-\bar{m}_{t}, e(\ell)\right)=u^{b}\left(0,0^{L}\right)$. By a similar argument as before, it holds that $\bar{m}_{t}$ is well-defined, uniquely determined, and greater than or equal to $\underline{p}_{\ell}$. Individual rationality of buyer $b$ implies that $\bar{m}_{t}$ is the highest amount of money that $b$ is ever going to pay for a contract involving trade $t$. If $\bar{p}_{\ell} \neq+\infty$, then either $u^{b}\left(-\bar{p}_{\ell}, e(\ell)\right) \geq u^{b}\left(0,0^{L}\right)$ and we define $\bar{m}_{t}=\bar{p}_{\ell}$, or $u^{b}\left(-\bar{p}_{\ell}, e(\ell)\right)<u^{b}\left(0,0^{L}\right)$ and we define $\bar{m}_{t} \in \mathbb{R}$ as the unique amount of money such that $u^{b}\left(-\bar{m}_{t}, e(\ell)\right)=u^{b}\left(0,0^{L}\right)$. By the same argument as before, $\bar{m}_{t}$ is well-defined, uniquely determined, and greater than or equal to $\underline{p}_{\ell}$. The upper bound on the price of commodity $\ell$, together with individual rationality of buyer $b$, implies that $\bar{m}_{t}$ is the highest amount of money that $b$ is ever going to pay for a contract involving trade $t$.

Next, we choose some $\varepsilon>0$ and define the finite set of contracts $\bar{Y}^{\varepsilon}$ by

$$
\bar{Y}^{\varepsilon}=\left\{(t, m) \in \bar{Y} \mid \exists k \in \mathbb{N}_{0} \text { such that } m=\min \left\{\underline{m}_{t}+k \varepsilon, \bar{m}_{t}\right\}\right\}
$$

In this way, only contracts $(t, m)$ are considered where the difference between $m$ and $\underline{m}_{t}$ is an integer multiple of $\varepsilon$ or $m=\bar{m}_{t}$. Moreover, this definition achieves that $m$ belongs to the interval $\left[\underline{m}_{t}, \bar{m}_{t}\right]$.

The adjustment process is now defined as follows.

\section{Definition 4.1: (Adjustment Process)}

Step 1: Initially, the set of permitted contracts $Y$ is equal to $\bar{Y}^{\varepsilon}$ and the set of tentatively accepted contracts $A$ is equal to $\emptyset$.

Step 2: Every buyer $b \in B \backslash \beta(A)$ chooses a unique member in the set $C^{b}(Y)$. Let $Z$ be the union of the set of contracts as chosen by $b \in B \backslash \beta(A)$ and the set $A$.

Step 3: Every seller $s \in \sigma(Z)$ tentatively accepts one arbitrarily chosen contract in $C^{s}(Z)$. Let $A$ be the set of contracts as tentatively accepted by $s \in \sigma(Z)$.

Step 4: The process stops if $A=Z$. In that case the contracts in $A$ are permanently accepted. Otherwise, the process returns to Step 2 with set of permitted contracts $Y \backslash(Z \backslash A)$. 
We illustrate the adjustment process for the case of the marriage problem. The adjustment process turns out to be identical to the deferred acceptance algorithm of Gale and Shapley (1962) in this case. For every choice of $\varepsilon>0$, it holds that

$$
\bar{Y}^{\varepsilon}=\bar{Y}=\{y \in B \times S \times(B \times S) \times\{0\} \mid \lambda(y)=(\beta(y), \sigma(y))\} .
$$

Step 1 defines the initial set of permitted contracts to be equal to $\bar{Y}$ and the set of tentatively accepted contracts to $\emptyset$. In Step 2, every man proposes to his favorite woman. In Step 3, every woman tentatively accepts her favorite man among those that have proposed to her and rejects all the others. The process stops in Step 4 if no man is rejected in Step 3 and all initial proposals become definite. Otherwise, the process returns to Step 2 with a set of permitted contracts where all the contracts rejected in Step 3 have been removed. In Step 2, the men that have been rejected now propose to their second choices. Every woman tentatively accepts her favorite man from the group consisting of the new proposers and the tentatively accepted man, if any. The others are rejected. The process continues in this way and will stop after finitely many iterations with a set of contracts where each man is matched to exactly one woman.

We next illustrate the adjustment process for the assignment model of Shapley and Shubik (1971). The adjustment process turns out to be identical to the approximate auction mechanism of Demange, Gale, and Sotomayor (1986) with personalized prices as discussed in Section $\mathrm{V}$ of that paper. For every $t=(b, s, \ell) \in T, \underline{m}_{t}=c_{s}$, the home owner's valuation of the house, and $\bar{m}_{t}=h_{b s}$, the valuation of the house of purchaser $b$. We take $\varepsilon=1$ and make use of the assumption that valuations are integer dollar amounts. It holds that

$$
\bar{Y}^{1}=\left\{(t, m) \in B \times S \times S \times \mathbb{Z} \mid \mu(t, m) \in\left[\underline{m}_{t}, \bar{m}_{t}\right]\right\}
$$

where $\mathbb{Z}$ denotes the set of integers. Step 1 defines the initial set of permitted contracts to be equal to $\bar{Y}^{1}$ and the set of tentatively accepted contracts to $\emptyset$. In Step 2, every purchaser $b$ maximizes $h_{b s}-c_{s}$ over all sellers $s$ and makes an offer $c_{s^{*}}$ to seller $s^{*}$ with $s^{*}$ one of his favorite sellers. In Step 3, every seller who receives one or more offers rejects all but one, which he tentatively accepts. Since a seller $s$ will initially only receive offers equal to $c_{s}$, he is completely indifferent among all offers received. The process stops in Step 4 if no buyer is rejected in Step 3 and all initial offers become definite. Otherwise, the process returns to Step 2 with a set of permitted contracts where all the contracts rejected in Step 3 have been removed. In Step 2, a purchaser $b$ that has been rejected now makes a new offer. There are several possibilities. For instance, if purchaser $b$ was indifferent between the selected seller $s^{*}$ in the previous round and exactly one other seller $s^{\prime}$, i.e. $h_{b s^{*}}-c_{s^{*}}=h_{b s^{\prime}}-c_{s^{\prime}}>0$, then he makes an offer of $c_{s^{\prime}}$ to seller $s^{\prime}$. If purchaser $b$ values seller $s^{*}$ at least two units more 
than the next best seller, i.e. $h_{b s^{*}}-c_{s^{*}}-2 \geq \max _{s \in S \backslash\left\{s^{*}\right\}}\left(h_{b s}-c_{s}\right)$ and $h_{b s^{*}}-c_{s^{*}} \geq 2$, then he makes an offer of $c_{s^{*}}+1$ to a seller $s^{*}$. In case of indifferences, the buyer makes any optimizing choice. Every seller tentatively accepts the best offer from the set consisting of the new offers and the tentatively accepted offer of the previous round, if any. The others are rejected. It is now no longer necessarily the case that all offers involve the same amount of money. The process continues in this way and will stop after finitely many iterations.

In case there are no ties, the adjustment process coincides with the generalized GaleShapley algorithm of Hatfield and Milgrom (2005), when starting with the set of contracts $\bar{Y}^{\varepsilon}$ for the buyers and the empty set of contracts for the sellers.

Talman and Yang (2008) define an adjustment process for the assignment model of Example 2.3, and Yang and Zhang (2013) show that this adjustment process terminates at a core allocation. The process is a variant of the Hungarian algorithm as used in the exact auction mechanism of Demange, Gale, and Sotomayor (1986) for the case without price controls. These processes are quite different from the one presented here. For instance, in each step of Demange, Gale, and Sotomayor (1986), price adjustments are made for minimal overdemanded sets of items. The determination of a minimal overdemanded set of items relies on the entire demand sets of all the purchasers and all purchasers report their entire demand set in each step of the process. There is also no such thing as a tentatively accepted offer.

A stable outcome $A$ for the set of feasible contracts $\bar{Y}^{\varepsilon}$ is defined as in Definition 3.1 with $\bar{Y}$ replaced by $\bar{Y}^{\varepsilon}$. This affects Definition 3.1 in two ways. First, the set of all outcomes $\mathcal{A}$ is defined using $\bar{Y}^{\varepsilon}$ instead of $\bar{Y}$, so all contracts in $A$ are required to belong to $\bar{Y}^{\varepsilon}$. Secondly, absence of blocking by a two-agent coalition as in Condition 2 of Definition 3.1 is restricted to absence of blocking by contracts in $\bar{Y}^{\varepsilon}$.

TheOREM 4.2: The adjustment process of Definition 4.1 terminates in a finite number of steps with a set $A$ of permanently accepted contracts. The set $A$ is a stable outcome for the set of feasible contracts $\bar{Y}^{\varepsilon}$.

\section{PROOF:}

Suppose the adjustment process does not terminate in a finite number of steps. Then, in Step 4 of each iteration, the set $Z \backslash A$ is non-empty and the cardinality of the set $Y$ in the next iteration diminishes by at least one. Since the initial set $Y$ as defined in Step 1 has finitely many elements, this leads to a contradiction.

Let $A$ be the set of permanently accepted contracts. Since by Step 2 every buyer is involved in at most one contract in $A$, and by Step 3 every seller is involved in at most one 
contract in $A$, it follows that $A$ is an outcome.

For every $(m, t) \in \bar{Y}^{\varepsilon}$, the definitions of $\underline{m}_{t}$ and $\bar{m}_{t}$ guarantee individual rationality for both the buyer and the seller involved in trade $t$. It follows that Condition 1 of Definition 3.1 is satisfied.

Consider the finite sequence of sets of tentatively accepted contracts as generated in the various iterations of the adjustment process and the associated finite sequence of utilities of a seller $s$. The finite sequence of utilities is weakly increasing at each iteration. Indeed, the first, if any, tentatively accepted contract by seller $s$ gives utility at least equal to $U^{s}\left(0,0^{L}\right)$ since all contracts in $\bar{Y}^{\varepsilon}$ are individual rational for seller $s$. Next, a seller can keep a tentatively accepted contract forever, or rejects it in favor of a contract that gives at least the same utility.

Suppose there is $z=(b, s, \ell, m) \in \bar{Y}^{\varepsilon}$ such that $U^{b}(\{z\})>U^{b}(A)$ and $U^{s}(\{z\})>U^{s}(A)$. Then, at some iteration of the adjustment process, $z$ has been rejected in Step 3 by seller $s$. The tentatively accepted contract by seller $s$ in that iteration, say $y$, is such that $U^{s}(\{y\}) \geq U^{s}(\{z\})$. Since the utility of the tentatively accepted contract by seller $s$ is weakly increasing at each iteration, it holds that $U^{s}(A) \geq U^{s}(\{y\}) \geq U^{s}(\{z\})$, leading to a contradiction. Consequently, Condition 2 of Definition 3.1 is satisfied.

We now turn to the existence of stable outcomes in the model without a smallest monetary unit.

Theorem 4.3: Consider an economy $\mathcal{E}=\left(T, \underline{p}, \bar{p},\left(u^{i}\right)_{i \in I}\right)$. A stable outcome exists.

\section{PROOF:}

Consider the sequence $\left(\varepsilon_{n}\right)_{n \in \mathbb{N}}$ with $\varepsilon_{n}=2^{-n}$ and let $A_{n}$ be the outcome that is generated by the adjustment process of Definition 4.1 for the set of feasible contracts $\bar{Y}^{\varepsilon_{n}}$. By listing the, potentially empty, contracts of the buyers, we can represent every $A_{n}$ as an element of the compact set

$$
(S \times L \times[\underline{m}, \bar{m}] \cup\{\emptyset\})^{B},
$$

where $\underline{m}$ is a finite lower bound for $\left\{\underline{m}_{t} \mid t \in T\right\}$ and $\bar{m}$ is a finite upper bound for $\left\{\bar{m}_{t} \mid\right.$ $t \in T\}$. The sequence $A_{n}$ therefore has a convergent subsequence, denoted by $\left(A_{n_{k}}\right)_{k \in \mathbb{N}}$, with limit, say, $A$.

The remainder of the proof verifies that $A$ is a stable outcome.

It clearly holds that $A \subset \bar{Y}$ is an outcome and that every contract $(t, m) \in A$ satisfies $\underline{m}_{t} \leq m \leq \bar{m}_{t}$. Condition 1 of Definition 3.1 corresponding to individual rationality is therefore satisfied by $A$. 
Suppose that there is $z=(b, s, \ell, m) \in \bar{Y}$ such that $U^{b}(\{z\})>U^{b}(A)$ and $U^{s}(\{z\})>$ $U^{s}(A)$. Since $A$ satisfies individual rationality, it follows that $\underline{m}_{b, s, \ell} \leq m \leq \bar{m}_{b, s, \ell}$. Then there is $n^{\prime} \in \mathbb{N}$ and $y=(b, s, \ell, \tilde{m}) \in \bar{Y}^{\varepsilon_{n^{\prime}}}$ such that $U^{b}(\{y\})>U^{b}(A)$ and $U^{s}(\{y\})>$ $U^{s}(A)$. Observe that, for every $n \geq n^{\prime}, y \in \bar{Y}^{\varepsilon_{n}}$.

Let $k^{\prime}$ be such that, for every $k \geq k^{\prime}, U^{b}(\{y\})>U^{b}\left(A_{n_{k}}\right)$ and $U^{s}(\{y\})>U^{s}\left(A_{n_{k}}\right)$. Let $k^{*}$ be such that $n_{k^{*}} \geq \max \left\{n^{\prime}, n_{k^{\prime}}\right\}$. It follows that $b$ and $s$ can use $y \in \bar{Y}^{\varepsilon_{k_{k^{*}}}}$ to block outcome $A_{n_{k^{*}}}$, thereby violating that $A_{n_{k^{*}}}$ is stable for the set of feasible contracts $\bar{Y}^{\varepsilon_{n^{*}}}$. Consequently, $A$ satisfies Condition 2 of Definition 3.2.

\section{$5 \quad$ Equilibrium Outcomes}

For markets in which all trading opportunities are universally available, it is natural to assume that the identity of the trading partner is irrelevant. In that case, it is customary to study notions of competitive equilibrium that assign a single price to each commodity, see, e.g., Gul and Stacchetti (1999) and Sun and Yang (2006). It is this perspective that we take in the current section, so we aim at developing a notion of competitive equilibrium for the economy $\mathcal{E}=\left(T, p, \bar{p},\left(u^{i}\right)_{i \in I}\right)$ as presented in Section 2, where each agent can trade against the same price vector. The initial endowment of every agent $i \in I$ is equal to $\left(0,0^{L}\right) \in X^{i}$.

For economies in which all commodities are perfectly divisible, there is an extensive literature on competitive equilibrium under price controls, starting with seminal contributions by Bénassy (1975), Drèze (1975), and Younès (1975). Herings (1996) provides an overview of this stream in the literature. Here, we follow the approach as developed in Drèze (1975) for economies where all commodities are divisible and extend this approach to the economy of Section 2.

In a competitive analysis of price controls, there is a price vector $p \in\{1\} \times \mathbb{R}^{L}$ against which trade in commodities takes place, with each commodity $\ell$ trading against a single price $p_{\ell}$. In the presence of a price floor $\underline{p}$ and a price ceiling $\bar{p}$, it should hold that $\underline{p}_{\ell} \leq p_{\ell} \leq$ $\bar{p}_{\ell}$ for every $\ell \in L$. A buyer $b \in B$ chooses a utility maximizing element $x^{b} \in X^{b}$ subject to the budget constraint $p x^{b} \leq 0$. Similarly, a seller $s \in S$ chooses a utility maximizing element $x^{s} \in X^{s}$ subject to the budget constraint $p x^{s} \leq 0$. Moreover, buyers and sellers may face rationing constraints to be explained below.

In case the price ceiling $\bar{p}_{\ell}$ is binding for some commodity $\ell$, its market may be cleared by quantity adjustments. Since prices are upward rigid, quantity adjustments are on the buyers' side. At a price equal to $\bar{p}_{\ell}$, a buyer might be willing to buy commodity $\ell$, but there is no seller willing to supply to him at that price. The price ceiling prevents the price 
to go up. Under such circumstances, a buyer faces demand rationing of commodity $\ell$.

Let $Q \subset\left\{(b, \ell) \in B \times L \mid \ell \in L^{b}\right\}$ be the set of demand rationing constraints. The collection of all such sets is denoted by $\mathcal{Q}$, so

$$
\mathcal{Q}=\left\{Q \in 2^{B \times L} \mid \text { for every }(b, \ell) \in Q, \ell \in L^{b}\right\} .
$$

If $(b, \ell) \in Q$, then buyer $b$ is rationed in his demand of commodity $\ell$, and when maximizing his utility, he takes into account the constraint $x_{\ell}^{b}=0$. The set $Q$ is determined endogenously in an equilibrium and could be equal to the empty set in which case no demand rationing is present. For $b \in B$, we define $Q^{b}=\{\ell \in L \mid(b, \ell) \in Q\}$ and for $\ell \in L$, we define $Q_{\ell}=\{b \in B \mid(b, \ell) \in Q\}$. The set $Q^{b}$ yields the commodities in which buyer $b$ is rationed in his demand. The set $Q_{\ell}$ contains the buyers which are rationed in their demand of commodity $\ell$.

In case the price floor $\underline{p}_{\ell}$ is binding for some commodity $\ell$, its market may be cleared by quantity adjustments. Since prices are downwards rigid, the quantity adjustments are on the sellers' side. Indeed, a seller may not face any demand for commodity $\ell$ when the price is $\underline{p}_{\ell}$, although he is willing to sell commodity $\ell$ against price $\underline{p}_{\ell}$. Moreover, the price floor prevents the price to go down. Under such circumstances, the seller faces supply rationing of commodity $\ell$.

Let $R \subset\left\{(s, \ell) \in S \times L \mid \ell \in L^{s}\right\}$ be the set of supply rationing constraints. The collection of all such sets is denoted by $\mathcal{R}$, so

$$
\mathcal{R}=\left\{R \in 2^{S \times L} \mid \text { for every }(s, \ell) \in R, \ell \in L^{s}\right\} .
$$

If $(s, \ell) \in R$, then seller $s$ is rationed in his supply of commodity $\ell$, and when maximizing his utility, he takes into account the constraint $x_{\ell}^{s}=0$. The set $R$ is determined endogenously in an equilibrium and could be equal to the empty set in which case no supply rationing is present. For $s \in S$, we define $R^{s}=\{\ell \in L \mid(s, \ell) \in R\}$ and for $\ell \in L$, we define $R_{\ell}=\{s \in S \mid(s, \ell) \in R\}$. The set $R^{s}$ yields the commodities in which seller $s$ is rationed in his supply. The set $R_{\ell}$ contains the sellers which are rationed in their supply of commodity $\ell$.

Given a price vector $p \in\{1\} \times \mathbb{R}^{L}$ and a set of rationing constraints $Q^{b} \subset L^{b}$, the budget set of a buyer $b \in B$ is given by

$$
\gamma^{b}\left(p, Q^{b}\right)=\left\{x^{b} \in X^{b} \mid p x^{b} \leq 0, \text { for every } \ell \in Q^{b}, x_{\ell}^{b}=0\right\} .
$$

The rationing constraints of buyers different from $b$ are irrelevant for the budget set of buyer $b$ and are therefore suppressed in the definition of $\gamma^{b}$. The demand correspondence $\delta^{b}$ of buyer $b$ is defined by

$$
\delta^{b}\left(p, Q^{b}\right)=\arg \max _{x^{b} \in \gamma^{b}\left(p, Q^{b}\right)} u^{b}\left(x^{b}\right) .
$$


The budget set $\gamma^{b}\left(p, Q^{b}\right)$ is non-empty as it contains $\left(0,0^{L}\right)$ as an element. It follows that $\delta^{b}\left(p, Q^{b}\right)$ is non-empty, since monotonicity of the utility function in money implies that the budget constraint can be defined with equality, and the maximization therefore takes place over a non-empty finite set of elements.

Given a price vector $p \in\{1\} \times \mathbb{R}^{L}$ and a set of rationing constraints $R^{s} \subset L^{s}$, the budget set of a seller $s \in S$ is given by

$$
\gamma^{s}\left(p, R^{s}\right)=\left\{x^{s} \in X^{s} \mid p x^{s} \leq 0, \text { for every } \ell \in R^{s}, x_{\ell}^{s}=0\right\}
$$

The rationing constraints of sellers different from $s$ are irrelevant for the budget set of seller $s$ and are therefore omitted in the definition of $\gamma^{s}$. The demand correspondence $\delta^{s}$ of seller $s$ is defined by

$$
\delta^{s}\left(p, R^{s}\right)=\arg \max _{x^{s} \in \gamma^{s}\left(p, R^{s}\right)} u^{s}\left(x^{s}\right) .
$$

Using the same argument as before, it follows that $\delta^{s}\left(p, R^{s}\right)$ is non-empty.

Definition 5.1: A Drèze equilibrium of the economy $\mathcal{E}=\left(T, \underline{p}, \bar{p},\left(u^{i}\right)_{i \in I}\right)$ is an element $\left(p^{*}, Q^{*}, R^{*}, x^{*}\right) \in\left(\{1\} \times \mathbb{R}^{L}\right) \times \mathcal{Q} \times \mathcal{R} \times X$ such that:

1. For every $b \in B, x^{* b} \in \delta^{b}\left(p^{*}, Q^{* b}\right)$.

2. For every $s \in S, x^{* s} \in \delta^{s}\left(p^{*}, R^{* s}\right)$.

3. $\sum_{i \in I} x^{* i}=\left(0,0^{L}\right)$.

4. For every $\ell \in L, \underline{p}_{\ell} \leq p_{\ell}^{*} \leq \bar{p}_{\ell}$.

5. For every $\ell \in L$, if $p_{\ell}^{*}<\bar{p}_{\ell}$, then $Q_{\ell}^{*}=\emptyset$.

6. For every $\ell \in L$, if $p_{\ell}^{*}>\underline{p}_{\ell}$, then $R_{\ell}^{*}=\emptyset$.

7. For every $\ell \in L, Q_{\ell}^{*}=\emptyset$ or $R_{\ell}^{*}=\emptyset$.

The first two conditions in Definition 5.1 reflect the standard optimizing behavior of the buyers and the sellers. Buyers and sellers need only know the given prices and their individual rationing scheme and need not consider the other individuals in making their choices. The third condition expresses market clearing. The fourth condition rules out exchange against prices violating the price controls. Condition 5 states that whenever the price of a commodity $\ell$ is upwards flexible, i.e. strictly below the price ceiling $\bar{p}_{\ell}$, then there is no rationing of the buyers. Instead, the standard price mechanism should result 
in higher prices when there is excess demand of commodity $\ell$. The price mechanism is prevented from clearing the market for commodity $\ell$ whenever the price $p_{\ell}^{*}$ is equal to $\bar{p}_{\ell}$ and there is excess demand of commodity $\ell$. In such a case, rationing of buyers may occur at equilibrium. Similarly, Condition 6 states that whenever the price of a commodity $\ell$ is downwards flexible, i.e. strictly above the price floor $\underline{p}_{\ell}$, then there is no rationing of the sellers. Instead, the standard price mechanism should result in lower prices when there is excess supply of commodity $\ell$. The price mechanism is prevented from clearing the market for commodity $\ell$ whenever the price $p_{\ell}^{*}$ is equal to $\underline{p}_{\ell}$ and there is excess supply of commodity $\ell$. In such a case, rationing of sellers may occur at equilibrium. Condition 7 expresses that markets are transparent. There is not simultaneously rationing on the supply and on the demand of a given commodity $\ell$.

When we apply Definition 5.1 to the case with only one commodity, the analysis reduces to the standard textbook analysis of the effects of price floors and price ceilings based on supply and demand curves. Perloff (2012), for instance, considers a price ceiling $\bar{p}$ on gasoline, a measure taken by the U.S. government in 1973 and 1979 in response to reduced supply of oil by OPEC. At $\bar{p}$, which is chosen below the market clearing price for gasoline, total demand for gasoline strictly exceeds total supply. The amount of gasoline demand which is fulfilled is exactly equal to the total supply of gasoline at $\bar{p}$, resulting in rationing of all the demand in excess of total supply at $\bar{p}$. This is exactly the same equilibrium as the Drèze equilibrium resulting from Definition 5.1, except that in Definition 5.1 it is also specified which buyers are going to be rationed. With a price ceiling on gasoline, but no price floor, Condition 6 of Definition 5.1 specifies that $R^{*}=\emptyset$, so there is no rationing on the supply side. Total supply of gasoline is therefore equal to the total number of sellers that are willing to supply at a price equal to $\bar{p}$. By Condition 3 of Definition 5.1, this will be equal to total demand for gasoline at a Drèze equilibrium. The needed reduction in demand for gasoline is achieved by rationing some of the buyers, so including them in $Q_{\ell}^{*}$. More precisely, the number of buyers facing binding demand rationing is exactly equal to the difference between total unrationed demand and total supply at $\bar{p}$. The amount of rationing of the long side of the market is determined by the short side.

There are no Drèze equilibria with a price below $\bar{p}$. At such prices demand rationing is ruled out by Condition 5. Since already at $\bar{p}$ total unrationed demand exceeds total supply, the same is true at prices lower than $\bar{p}$, and market clearing is not possible. Unlike Definition 5.1, the standard textbook analysis does not take into account that other markets are going to be affected by the price ceiling on gasoline, and ignores all general equilibrium effects.

When we apply Definition 5.1 to the marriage market, the set $Q^{b}$ specifies all the women that are unavailable for man $b$, and, similarly, $R^{s}$ specifies all the men that are not available 
for woman $s$. Condition 4 states that no monetary transfers are made. Conditions 5 and 6 are vacuous. By Condition 7 it is ruled out at equilibrium that there is a pair $(b, s)$ of a man and a woman such that simultaneously $(b, s) \in Q^{b}$ and $(b, s) \in R^{s}$. It holds that woman $s$ can be proposed to by man $b$ or that man $b$ can be proposed to by woman $s$. Condition 1 now expresses that all men choose the best available woman and Condition 2 that all women choose the best available man. If a man or a woman does not face any rationing constraints, then he or she is free to choose the best possible partner. Condition 3 , market clearing, is now equivalent to the requirement that if man $b$ chooses woman $s$, then also woman $s$ chooses man $b$, and vice versa. Rationing of men and women is determined endogenously at equilibrium and is a consequence of men or women being in excess demand of the opposite sex.

Definition 5.1 extends the equilibrium concept as developed in Drèze (1975). Drèze's concept has been defined for economies satisfying the standard assumptions of traditional general equilibrium analysis with divisible commodities, thereby ruling out the discrete matching with contracts case as studied in this paper.

In the absence of price controls, so when, for every $\ell \in L, \underline{p}_{\ell}=-\infty$ and $\bar{p}_{\ell}=+\infty$, Definition 5.1 reduces to the standard definition of a Walrasian equilibrium. It follows immediately from Conditions 5 and 6 that $Q^{*}=R^{*}=\emptyset$, so Condition 7 is automatically satisfied. Condition 4 becomes void. What remains are the two standard Conditions 1 and 2, expressing optimization without rationing constraints, and the market clearing Condition 3.

For the assignment model with price controls and quasi-linear utility functions as presented in Example 2.3, Talman and Yang (2008) define the concept of constrained Walrasian equilibrium. It is not difficult to show that the application of the Drèze equilibrium of Definition 5.1 to the economy of Example 2.3 coincides with the constrained Walrasian equilibrium.

Andersson and Svensson (2014) consider the model of house allocation with price controls of Example 2.4. They propose the concept of a rationing price equilibrium and specify an allocation rule that always selects a rationing price equilibrium. The allocation rule is shown to be constrained efficient and non-manipulable for almost all preference profiles. For the extreme cases in which rents are fully flexible or completely fixed, the allocation rule coincides with the one from the competitive price mechanism of Demange and Gale (1985) and the deferred acceptance algorithm of Gale and Shapley (1962), respectively. The rationing price equilibrium is a Drèze equilibrium, but not vice versa. Indeed, a rationing price equilibrium satisfies by definition a particular constrained efficiency criterion, which is closely related to Pareto efficiency from the buyers' point of view. In the absence of price rigidities, this boils down to selecting the competitive equilibrium at minimum 
Walrasian prices, whereas Definition 5.1 would comprise all competitive equilibria. Andersson and Svensson (2014) also require that rationing constraints correspond to some given priorities, whereas Definition 5.1 allows for all rationing constraints compatible with the seven conditions.

Our next example illustrates the concept of Drèze equilibrium for the model of quality competition in the presence of price controls of Example 2.5. We consider again the economy with primitives derived from Table 1 and consider a uniform price floor $f$ equal to 8.5 for all qualities. Since at prices greater than or equal to $f$, buyers $b_{2}$ and $b_{3}$ always express a demand of $\left(0,0^{L}\right)$, the economy essentially reduces to an economy with one buyer, $b_{1}$, and three sellers.

Let $\left(p^{*}, Q^{*}, R^{*}, x^{*}\right)$ be a Drèze equilibrium. Since price ceilings are equal to $+\infty$ for all qualities, Condition 5 specifies that $Q^{*}=\emptyset$. Condition 7 of Definition 5.1 is then trivially satisfied.

Suppose $p_{1}^{*}>8.5$. Since $p_{1}^{*}>f$, there is no rationing of quality 1 according to Condition 6 of Definition 5.1. Every seller strictly prefers selling quality 1 to being inactive. Total supply of all qualities together is then equal to 3 . Since buyers $b_{2}$ and $b_{3}$ are not willing to buy any quality at prices greater than or equal to 8.5, total demand of qualities is less than or equal to 1. This violates the market clearing Condition 3 of Definition 5.1. Consequently, it holds that $p_{1}^{*}=8.5$. By exactly the same argument, it follows that $p_{2}^{*}=8.5$.

There are two possibilities for $p_{3}^{*}$. It holds that either $p_{3}^{*}>8.5$ or $p_{3}^{*}=8.5$. In the former case, $R_{3}^{*}=\emptyset$ and total supply of qualities is at least two units, which strictly exceeds total demand of qualities, being equal to at most one unit. We obtain $p_{3}^{*}=8.5$. Since there is no demand for qualities 1 and 2 when $p_{1}^{*}=8.5$, whereas all sellers prefer the supply of qualities 1 and 2 to the supply of quality 3, Condition 3 of Definition 5.1 implies that $R_{1}^{*}=R_{2}^{*}=\left\{s_{1}, s_{2}, s_{3}\right\}$.

Essentially, there are two Drèze equilibria. In both equilibria, buyer $b_{1}$ demands one unit of quality 3. In one equilibrium, seller $s_{1}$ supplies one unit of quality 3 against a price $p_{3}^{*}=8.5$ and $s_{2} \in R_{3}^{*}$, and in the other equilibrium seller $s_{2}$ supplies one unit of quality 3 against a price $p_{3}^{*}=8.5$ and $s_{1} \in R_{3}^{*}$. Whether $s_{3}$ belongs to $R_{3}^{*}$ or not is irrelevant, since the cost of producing quality 3 is equal to 9 for $s_{3}$, so exceeds $p_{3}^{*}$ and there is no supply of quality 3 by seller $s_{3}$ in either case.

In both equilibria there are trades with a positive surplus which are not carried out, since they require a price below the price floor. Also, the partners involved in the single equilibrium trade would both benefit from trading a lower quality against a price below the price floor. The equilibrium where quality 3 is supplied to $b_{1}$ by $s_{2}$ is not even constrained efficient, as the equilibrium where quality 3 is supplied to $b_{1}$ by $s_{1}$ leads to a strictly higher sum of utilities. The equilibrium where quality 3 is supplied to $b_{1}$ by $s_{1}$ is constrained 
efficient.

It is well-documented in the literature with divisible commodities that price controls and price regulations lead to various kinds of inefficiencies. Bulow and Klemperer (2012) show in a partial equilibrium context that price controls always reduce consumer surplus when demand is convex and less elastic than supply, or when demand is log convex irrespective of the elasticity of supply. Herings and Konovalov (2009) demonstrate in a general equilibrium context that under mild conditions price controls induce allocations that are conducive to Pareto improvements even when respecting the price controls. Such a result cannot be extended in a straightforward way to the context of this paper. The Drèze equilibrium where quality 3 is supplied to $b_{1}$ by $s_{1}$ is constrained efficient and would remain so after small perturbations to the underlying economy.

\section{Equivalence of Stable Outcomes and Drèze Equilib- ria}

Shapley and Shubik (1971) consider the assignment game and prove that the set of stable payoffs coincides with the set of competitive equilibrium payoffs. This relationship between stable outcomes and competitive equilibria has been substantially generalized, most notably in the direction of set-ups with many-to-many matching or even trading networks as demonstrated by Hatfield, Kominers, Nichifor, Ostrovsky, and Westkamp (2013). These results are invariably derived in a setting with unlimited monetary transfers, or, equivalently, absence of price controls. In this section, we obtain a fully general equivalence between stable outcomes and Drèze equilibria. This equivalence boils down to the equivalence with competitive equilibria in the absence of price controls, presents a completely new result for cases with no monetary transfers like the marriage problem, and also deals with all intermediate cases like wage or rent controls.

The concepts of stable outcome and Drèze equilibrium are not directly comparable. On the one hand, a stable outcome provides more information, as it is specified who trades with whom. On the other hand, in a Drèze equilibrium also the prices of commodities that are not traded are specified and a Drèze equilibrium specifies explicit rationing constraints.

Although it is not possible to directly compare a stable outcome to a Drèze equilibrium, we can compare the resulting allocations. In fact, this is all that matters from a welfare point of view.

Consider an economy $\mathcal{E}=\left(T, \underline{p}, \bar{p},\left(u^{i}\right)_{i \in I}\right)$. The set of allocations corresponding to stable outcomes is $X^{\text {so }}$,

$$
X^{\text {so }}=\{x \in X \mid \text { there is a stable outcome } A \text { such that } \xi(A)=x\} .
$$


The set of Drèze equilibrium allocations is $X^{\mathrm{D}}$,

$X^{\mathrm{D}}=\left\{x^{*} \in X \mid\right.$ there is $\left(p^{*}, Q^{*}, R^{*}\right)$ such that $\left(p^{*}, Q^{*}, R^{*}, x^{*}\right)$ is a Drèze equilibrium $\}$. The next result shows that if $A$ is a stable outcome, then there is a Drèze equilibrium with allocation $\xi(A)$.

Theorem 6.1: Consider an economy $\mathcal{E}=\left(T, p, \bar{p},\left(u^{i}\right)_{i \in I}\right)$. It holds that $X^{\text {so }} \subset X^{\mathrm{D}}$.

Proof: See Appendix.

Consider some stable outcome $A$. Step 1 in the proof shows that each commodity in $\lambda(A)$ trades at a single price. Indeed, for every $y, y^{\prime} \in A$ such that $\lambda(y)=\lambda\left(y^{\prime}\right)$ it holds that $\mu(y)=\mu\left(y^{\prime}\right)$. In the quality competition model, for instance, a single quality cannot be part of two contracts involving different amounts of money. Intuitively, whenever two contracts involve different amounts of money, the buyer paying the high amount wants to match with the seller receiving the low amount, and write a contract with an amount of money somewhere in the middle. Since $T$ has a product structure, such a match is feasible. This makes it possible to use the stable outcome $A$ in the construction of a Drèze equilibrium, where a commodity trades against a single price by definition. The price of a commodity $\ell \in \lambda(A)$ is denoted by $p_{\ell}^{*}$.

For $\ell \in L$ and $b \in \beta\left(\bar{Y}^{\ell}\right)$, the extended real number $r_{\ell}^{b}$ denotes the reservation value of commodity $\ell$ for buyer $b$. By definition, it is equal to the value of $m$ for which $u^{b}(-m, e(\ell))=$ $u^{b}\left(x^{* b}\right)$, where $x^{* b}$ is the consumption bundle of buyer $b$ as induced by the stable outcome $A{ }^{1}$ The highest reservation value among all buyers in $\beta\left(\bar{Y}^{\ell}\right)$ is denoted by $r_{\ell}^{B}$. Similarly, for $\ell \in L$ and $s \in \sigma\left(\bar{Y}^{\ell}\right), r_{\ell}^{s}$ denotes the reservation value of commodity $\ell$ for seller $s$, so $u^{s}\left(r_{\ell}^{s},-e(\ell)\right)=u^{s}\left(x^{* s}\right)$, and $r_{\ell}^{S}$ denotes the lowest reservation value among all sellers in $\sigma\left(\bar{Y}^{\ell}\right)$.

In Step 3 of the proof of Theorem 6.1 it is shown that for any traded commodity $\ell \in \lambda(A)$ at least one of three cases holds. The first possibility is that the price $p_{\ell}^{*}$ against which commodity $\ell$ is traded is equal to the price floor $\underline{p}_{\ell}$, the lowest reservation value among sellers $r_{\ell}^{S}$ is less than or equal to $\underline{p}_{\ell}$, and the highest reservation value among buyers $r_{\ell}^{B}$ is equal to $\underline{p}_{\ell}$. In this case, a seller $s$ may have a reservation value $r_{\ell}^{s}<\underline{p}_{\ell}$, but the price floor $\underline{p}_{\ell}$ prevents a price decrease and the seller is rationed on his supply of commodity $\ell$. Since none of the buyers $b$ has a reservation value $r_{\ell}^{b}>p_{\ell}^{*}$, there is no need for rationing buyers on their demand of commodity $\ell$.

The second possibility as described in Step 3 of the proof of Theorem 6.1 is that the price $p_{\ell}^{*}$ against which commodity $\ell$ is traded is strictly in between the price floor $\underline{p}_{\ell}$ and

\footnotetext{
${ }^{1}$ In the proof, we also deal carefully with the cases where $u^{b}(-m, e(\ell))<u^{b}\left(x^{* b}\right)$ for all $m \in \mathbb{R}$ and $u^{b}(-m, e(\ell))>u^{b}\left(x^{* b}\right)$ for all $m \in \mathbb{R}$.
} 
the price ceiling $\bar{p}_{\ell}$, and equal to the lowest reservation value among sellers $r_{\ell}^{S}$ and the highest reservation value among buyers $r_{\ell}^{B}$. This case corresponds to a market without effective price controls and absence of rationing. It will always occur when the price floor $\underline{p}_{\ell}$ is equal to $-\infty$ and the price ceiling $\bar{p}_{\ell}$ is equal to $+\infty$.

The third possibility of Step 3 parallels the first case, with the role of buyers replaced by sellers. The price $p_{\ell}^{*}$ against which commodity $\ell$ is traded is equal to the price ceiling $\bar{p}_{\ell}$, the lowest reservation value among sellers $r_{\ell}^{S}$ is equal to $\bar{p}_{\ell}$, and the highest reservation value among buyers $r_{\ell}^{B}$ is greater than or equal to $\bar{p}_{\ell}$. In this case, a buyer $b$ may have a reservation value $r_{\ell}^{b}>\bar{p}_{\ell}$, but the price ceiling $\bar{p}_{\ell}$ prevents a price increase and the buyer is rationed on his demand of commodity $\ell$. Since none of the sellers $s$ has a reservation value $r_{\ell}^{s}<p_{\ell}^{*}$, there is no need for rationing sellers on their supply of commodity $\ell$.

In degenerate cases, the first and the third possibility can occur simultaneously. In this case the price floor must be equal to the price ceiling, the lowest reservation value among sellers, and the highest reservation value among buyers. The behavior of the market resembles the one of the second possibility. There are no effective price controls and there is absence of rationing.

In Step 4 of the proof of Theorem 6.1 it is shown that for any non-traded commodity $\ell \in L \backslash \lambda(A)$ at least one of three cases holds. The first possibility is that the highest reservation value $r_{\ell}^{B}$ among buyers for commodity $\ell$ is less than or equal to $\underline{p}_{\ell}$. In that case, the price floor is so high that all potential trade in commodity $\ell$ is killed off. The lowest reservation value among sellers of commodity $\ell$ may be higher or lower than $r_{\ell}^{B}$. In case it is lower, welfare gains could be achieved by contracts among such a seller and a buyer with a strictly higher reservation value. Since $r_{\ell}^{S}$ can be strictly below the price floor $\underline{p}_{\ell}$, supply rationing of sellers may occur in equilibrium. Since $r_{\ell}^{B} \leq \underline{p}_{\ell}$, there will be no simultaneous rationing of the demand of buyers. The equilibrium price $p_{\ell}^{*}$ of such a non-traded commodity can be set equal to $\underline{p}_{\ell}$ if $\underline{p}_{\ell}>-\infty$. If $\underline{p}_{\ell}=-\infty$, and therefore $r_{\ell}^{B}=-\infty$, so none of the buyers have an interest to acquire commodity $\ell$ against any price, $p_{\ell}^{*}$ can be set equal to any value less than or equal to the price ceiling $\bar{p}_{\ell}$ and the lowest reservation value among all sellers $r_{\ell}^{S}$.

The second possibility in Step 4 of the proof of Theorem 6.1 concerns the case where the highest reservation value among buyers $r_{\ell}^{B}$ is strictly above the price floor $\underline{p}_{\ell}$ and the lowest reservation value among sellers $r_{\ell}^{S}$ is strictly below the price ceiling $\bar{p}_{\ell}$, and, moreover, $r_{\ell}^{B}<r_{\ell}^{S}$. The market for such a commodity resembles a market without price controls and does not involve any rationing. Commodity $\ell$ does simply not admit a profitable trade among a buyer and a seller in this case. The equilibrium price of such a commodity can be any value in between $r_{\ell}^{B}$ and $r_{\ell}^{S}$.

The third possibility of Step 4 is the analogue of the first possibility with buyers replaced 
by sellers. The lowest reservation value $r_{\ell}^{S}$ among sellers of commodity $\ell$ is greater than or equal to $\bar{p}_{\ell}$. The price ceiling is so low that all potential supply of commodity $\ell$ is wiped out. If the highest reservation value among buyers exceeds $\bar{p}_{\ell}$, then demand rationing of such buyers occurs. The equilibrium price of such a commodity is taken equal to $\bar{p}_{\ell}$ if $\bar{p}_{\ell}<+\infty$ and equal to any value greater than or equal to $\underline{p}_{\ell}$ and $r_{\ell}^{B}$ otherwise.

The first and third possibility of Step 4 can occur simultaneously, also in non-degenerate cases. In this case, the market for commodity $\ell$ is characterized by the absence of any supply and demand.

Theorem 4.3 asserts that a stable outcome exists. Using Theorem 6.1, we can then infer the existence of a Drèze equilibrium as a corollary.

Corollary 6.2: Consider an economy $\mathcal{E}=\left(T, \underline{p}, \bar{p},\left(u^{i}\right)_{i \in I}\right)$. A Drèze equilibrium exists.

The next result presents the converse of Theorem 6.1. If $\left(p^{*}, Q^{*}, R^{*}, x^{*}\right)$ is a Drèze equilibrium, then there is a stable outcome $A$ such that $\xi(A)=x^{*}$.

Theorem 6.3: Consider an economy $\mathcal{E}=\left(T, \underline{p}, \bar{p},\left(u^{i}\right)_{i \in I}\right)$. It holds that $X^{\mathrm{D}} \subset X^{\text {so }}$.

\section{PROOF:}

Step 1. Definition of a stable outcome.

By Corollary 6.2, the set $X^{D}$ is non-empty. Take some $x^{*} \in X^{\mathrm{D}}$ and choose $\left(p^{*}, Q^{*}, R^{*}\right) \in$ $\left(\{1\} \times \mathbb{R}^{L}\right) \times \mathcal{Q} \times \mathcal{R}$ such that $\left(p^{*}, Q^{*}, R^{*}, x^{*}\right)$ is a Drèze equilibrium. For every $\ell \in L$ with $x_{\ell}^{*} \neq 0$, Condition 3 of Definition 5.1 implies that there are exactly as many buyers as sellers. Match every buyer $b \in B$ with $x_{\ell}^{* b}=1$ to a seller $s(b) \in S$ with $x_{\ell}^{*(b)}=-1$, so in particular $b \neq b^{\prime}$ implies $s(b) \neq s\left(b^{\prime}\right)$, and define a contract $y^{b}=\left(b, s(b), \ell, p_{\ell}^{*}\right)$. We define $A$ as the set of all such contracts, $A=\cup_{\left\{b \in B \mid x^{* b} \neq\left(0,0^{L}\right)\right\}}\left\{y^{b}\right\}$. It clearly holds that, for every $i \in I, U^{i}(A)=u^{i}\left(x^{* i}\right)$, and $\xi(A)=x^{*}$.

Step 2. For every $i \in I, A^{i} \in C^{i}(A)$.

Suppose for some $b \in B, A^{b} \notin C^{b}(A)$, so $u^{b}\left(0,0^{L}\right)>u^{b}\left(x^{* b}\right)$, a contradiction to $x^{* b} \in$ $\delta^{b}\left(p^{*}, Q^{* b}\right)$. Consequently, it holds for every $b \in B$ that $A^{b} \in C^{b}(A)$.

Suppose for some $s \in S, A^{s} \notin C^{s}(A)$, so $u^{s}\left(0,0^{L}\right)>u^{s}\left(x^{* s}\right)$, a contradiction to $x^{* s} \in$ $\delta^{s}\left(p^{*}, R^{* s}\right)$. Consequently, it holds for every $s \in S$ that $A^{s} \in C^{s}(A)$. 
It follows that Condition 1 of Definition 3.1 is satisfied.

Step 3. For every $z \in \bar{Y}$ it holds that $U^{b}(\{z\}) \leq U^{b}(A)$ or $U^{s}(\{z\}) \leq U^{s}(A)$.

Suppose there is $z=(b, s, \ell, m) \in \bar{Y}$ such that $U^{b}(\{z\})>U^{b}(A)=u^{b}\left(x^{* b}\right)$ and $U^{s}(\{z\})>U^{s}(A)=u^{s}\left(x^{* s}\right)$. By Condition 7 of Definition 5.1, it holds that $Q_{\ell}^{*}=\emptyset$ or $R_{\ell}^{*}=\emptyset$.

Consider the case with $Q_{\ell}^{*}=\emptyset$ first. Since $x^{* b} \in \delta^{b}\left(p^{*}, Q^{* b}\right)$ and $U^{b}(\{z\})>u^{b}\left(x^{* b}\right)$, it holds that $(-m, e(\ell)) \notin \gamma^{b}\left(p^{*}, Q^{* b}\right)$. Since there is no demand rationing of commodity $\ell$, it now follows that $m<p_{\ell}^{*}$. Since $z \in \bar{Y}$, this implies in turn that $\underline{p}_{\ell}<p_{\ell}^{*}$, so, according to Condition 6 of Definition 5.1, $R_{\ell}^{*}=\emptyset$. Since there is no supply rationing of commodity $\ell$, $U^{s}(\{z\})>u^{s}\left(x^{* s}\right)$ implies that $m>p_{\ell}^{*}$, and we have obtained a contradiction.

Consider next the case with $R_{\ell}^{*}=\emptyset$. Since $x^{* s} \in \delta^{s}\left(p^{*}, R^{* s}\right)$ and $U^{s}(\{z\})>u^{s}\left(x^{* s}\right)$, it holds that $(m,-e(\ell)) \notin \gamma^{s}\left(p^{*}, R^{* s}\right)$. Since there is no supply rationing of commodity $\ell$, it now follows that $m>p_{\ell}^{*}$. Since $z \in \bar{Y}$, this implies in turn that $\bar{p}_{\ell}>p_{\ell}^{*}$, so, according to Condition 5 of Definition 5.1, $Q_{\ell}^{*}=\emptyset$. Since there is no demand rationing of commodity $\ell$, $U^{b}(\{z\})>u^{b}\left(x^{* b}\right)$ implies that $m<p_{\ell}^{*}$, and again we have obtained a contradiction.

It follows that Condition 2 of Definition 3.1 is satisfied.

The outcome $A$ is stable and therefore $\xi(A)=x^{*} \in X^{\text {so }}$.

Combining the results of Theorem 6.1 and Theorem 6.3 leads to the following corollary.

Corollary 6.4: Consider an economy $\mathcal{E}=\left(T, \underline{p}, \bar{p},\left(u^{i}\right)_{i \in I}\right)$. It holds that $X^{\text {so }}=X^{\mathrm{D}}$.

It is not hard to understand the equivalence of Corollary 6.4 for the marriage problem of Example 2.1. Consider some stable outcome. For every $(b, s) \in B \times S$, it holds that $p_{b, s}^{*}=0$. For each man $b$, let $Q_{b}^{*}$ be the set of all women that are strictly preferred by $b$ to the woman matched to $b$ in the stable outcome. Similarly, for each woman $s$, let $R_{s}^{*}$ be the set of all men that are strictly preferred by $s$ to the man matched to $s$ in the stable outcome. These rationing constraints lead to sets of feasible partners for all men and women. Demand and supply of men and women will single out the partner as assigned in the stable outcome. Market clearing is obvious. It remains to be verified that there is no $(b, s) \in B \times S$ such that $(b, s) \in Q_{b}^{*}$ and $(b, s) \in R_{s}^{*}$. By definition of $Q_{b}^{*}$ and $R_{s}^{*}$, this would mean that $s$ is strictly preferred by $b$ to his match in the stable outcome and $b$ is strictly preferred by $s$ to her match in the stable outcome, and would contradict that we were starting from a stable outcome.

Consider some Drèze equilibrium for the marriage problem. The equilibrium allocation 
provides a unique matching between the men and the women and, since there are no monetary transfers, a unique outcome. Suppose the outcome is not stable. Then there is $(b, s) \in B \times S$ such that $b$ strictly prefers $s$ to his match in the outcome, and $s$ strictly prefers $b$ to her match in the outcome. Since the outcome is induced by the equilibrium allocation, which is in turn based on demand and supply, it follows that $(b, s) \in Q_{b}^{*}$ and $(b, s) \in R_{s}^{*}$, violating Condition 7 of Definition 5.1.

The matching concept of stability and the market equilibrium concept of Drèze equilibrium lead to exactly the same conclusions as far as allocations are concerned. The result is true in a set-up with unrestricted monetary transfers, thereby generalizing the equivalence of stable outcomes in the assignment model of Shapley and Shubik (1971) and Walrasian equilibrium outcomes to the matching with contracts set-up of this paper. The result holds in a set-up without any monetary transfers, so it presents an equivalence between stable outcomes in the marriage problem of Gale and Shapley (1962) and an appropriately specified market equilibrium concept. The result is valid in a set-up with limited monetary transfers, so for models as considered in Talman and Yang (2008), Hatfield, Plott, and Tanaka (2012b) and Andersson and Svensson (2014) the set of stable outcomes is equivalent to the set of Drèze equilibria.

\section{Conclusion}

This paper considers a discrete matching with contracts framework where contracts are subject to price controls. The framework is sufficiently general to admit many important models as special cases, like for instance the marriage problem of Gale and Shapley (1962), the assignment model without price controls of Shapley and Shubik (1971), the assignment model subject to price controls as in Talman and Yang (2008) or Andersson and Svensson (2014), and the quality competition model subject to price controls of Hatfield, Plott, and Tanaka (2012b).

The paper presents an adjustment process that always terminates with a stable outcome for the discretized version of the model. For the marriage problem, the adjustment process coincides with the deferred acceptance algorithm, for the assignment model without price controls, the adjustment process coincides with the approximate auction mechanism as discussed in Section V of Demange, Gale, and Sotomayor (1986), and in the case without ties, the adjustment process coincides with the generalized Gale-Shapley algorithm of Hatfield and Milgrom (2005). A limit argument can now be used to demonstrate the existence of a stable outcome in a model without a smallest monetary unit.

The paper proceeds by developing an equilibrium concept for the framework under consideration. In the presence of price floors and price ceilings, rationing may be needed to 
clear the markets. In the equilibrium approach, rationing constraints cannot be arbitrary. Rationing on the demand side is only allowed in the presence of price ceilings and rationing on the demand side only in the presence of price floors. Rationing on supply and demand cannot occur simultaneously in a given market: markets are transparent. Buyers and sellers take prices and rationing constraints as given and maximize their utility given these constraints. At equilibrium, prices and rationing schemes are endogenously determined to equalize supply and demand of all commodities. The equilibrium concept is an extension of the concept of Drèze (1975) as developed for economies with divisible commodities. The properties of the model with divisible commodities and the discrete set-up considered here are not the same. For instance, in the model with divisible commodities, under weak assumptions, equilibria are not constrained efficient. In the model with indivisible commodities, robust examples with constrained efficient equilibria can be constructed.

The relationship between solution concepts coming from matching theory and those resulting from competitive analysis is an important topic of research. This paper contributes to this literature by showing that the set of stable allocations according to the matching approach is the same as the set of Drèze equilibrium allocations in the presence of price controls like price floors or price ceilings. Since price floors can be set equal to minus infinity and price ceilings to plus infinity, the case with absence of price floors and price ceilings is a special case. In this case, Drèze equilibria are equivalent to Walrasian equilibria and the result specializes to the equivalence between Walrasian equilibria and stable outcomes. Since price floors and price ceilings can be both set equal to zero, the case without monetary transfers is a special case as well. As an example, the set of Drèze equilibria leads to a set of allocations that is equal to the one induced by stable outcomes in the marriage problem. Finally, there are many cases of interest in between unlimited monetary transfers and absence thereof, for instance, when studying the effects of minimum wages or rent controls. It is comfortable to know that the matching approach and the equilibrium approach lead to equivalent predictions in these cases as well.

There are at least three important generalizations of the current model where the theory of competitive equilibrium with price rigidities could benefit from the matching approach and where the question of equivalence between the approaches should be addressed. One generalization is the extension to the case of many-to-many matching or the case where a network of agents can trade indivisible commodities as in Hatfield, Kominers, Nichifor, and Westkamp (2013). Already in the case of many-to-one matching, the condition that the set of traders in a commodity has a product structure is not sufficient to guarantee that a single commodity trades against a single price. One way to establish the equivalence result in that case is to use personalized prices. Hatfield and Kominers (2012) show that without continuous transfers, in markets that lack a vertical structure, stable outcomes may not 
exist. This suggests that results with price controls may not generalize so easily beyond a-cyclical cases.

Price controls are often thought of as being a response to market failures like market incompleteness, see Polemarchakis (1979) and in particular Drèze and Gollier (1993). The main idea here is that in an incomplete markets set-up, fixed prices may offer insurance against risks that is not provided by flexible price contracts. Herings and Polemarchakis (2005) show that in an incomplete markets world it is generically possible to make Pareto improvements by choosing appropriate price regulations. The question is then whether the matching analysis can be extended to the incomplete markets case and whether in the presence of market incompleteness, contracts with fixed prices could outperform contracts with flexible prices.

Another reason to be interested in price rigidities is the observation that many commodity prices are sticky. Based on a detailed analysis on the distribution of the frequency of price changes in Nakamura and Steinsson (2008), Nakamura and Steinsson (2010) report that the median frequency of monthly price change across sectors in the U.S. economy is $8.7 \%$, implying that the median duration of a particular price across sectors is around one year. Citanna, Crès, Drèze, Herings, and Villanacci (2001) show that the presence of price stickiness gives rise to coordination failures and multiplicity of equilibria. The introduction of fiat money in general equilibrium models with price stickiness along the lines as suggested by modern monetary theory as presented in Woodford (2003), results in a high degree of real indeterminacy of equilibrium as is shown by Herings (2014). The question is then whether in an extension of the matching approach allowing for fiat money this indeterminacy still prevails and sharp predictions about the effects of monetary policy on equilibrium outcomes are not possible. 


\section{Appendix: Proof of Theorem 6.1}

Theorem 6.1: Consider an economy $\mathcal{E}=\left(T, \underline{p}, \bar{p},\left(u^{i}\right)_{i \in I}\right)$. It holds that $X^{\text {so }} \subset X^{\mathrm{D}}$.

Proof: Consider some $x^{*} \in X^{\text {so }}$ and let $A$ be a stable outcome such that $\xi(A)=x^{*}$. We prove the result in ten steps.

Step 1. A commodity trades at a single price: For every $y, y^{\prime} \in A$ such that $\lambda(y)=\lambda\left(y^{\prime}\right)$ it holds that $\mu(y)=\mu\left(y^{\prime}\right)$.

Suppose $\mu(y) \neq \mu\left(y^{\prime}\right)$ and without loss of generality assume $\mu(y)<\mu\left(y^{\prime}\right)$. Consider the contract

$$
z=\left(b, s, \lambda(y), \frac{1}{2} \mu(y)+\frac{1}{2} \mu\left(y^{\prime}\right)\right)
$$

where $b=\beta\left(y^{\prime}\right)$ and $s=\sigma(y)$. Since

$$
\underline{p}_{\lambda(y)} \leq \mu(y)<\frac{1}{2} \mu(y)+\frac{1}{2} \mu\left(y^{\prime}\right)<\mu\left(y^{\prime}\right) \leq \bar{p}_{\lambda(y)},
$$

and the set of traders in commodity $\ell$ has a product structure, it holds that $z \in \bar{Y}$. The utilities corresponding to $z$ are equal to

$$
\begin{aligned}
& U^{b}(\{z\})=u^{b}\left(-\frac{1}{2} \mu(y)-\frac{1}{2} \mu\left(y^{\prime}\right), e(\lambda(y))\right)>u^{b}\left(-\mu\left(y^{\prime}\right), e\left(\lambda\left(y^{\prime}\right)\right)\right)=U^{b}(A), \\
& U^{s}(\{z\})=u^{s}\left(\frac{1}{2} \mu(y)+\frac{1}{2} \mu\left(y^{\prime}\right),-e(\lambda(y))\right)>u^{s}(\mu(y),-e(\lambda(y)))=U^{s}(A),
\end{aligned}
$$

so Condition 2 of Definition 3.1 is violated and the outcome $A$ is not stable, a contradiction. Consequently, for every $y, y^{\prime} \in A$ such that $\lambda(y)=\lambda\left(y^{\prime}\right)$ it holds that $\mu(y)=\mu\left(y^{\prime}\right)$.

Step 2. Definition of prices for traded commodities and reservation values for all commodities.

For every $\ell \in \lambda(A)$, we denote the price at which $\ell$ is traded by $p_{\ell}^{*}$, so for every $y \in A$ such that $\lambda(y)=\ell$ it holds that $\mu(y)=p_{\ell}^{*}$.

Consider some $\ell \in L$ and some $b \in \beta\left(\bar{Y}^{\ell}\right)$. If, for every $m \in \mathbb{R}, u^{b}(-m, e(\ell))>u^{b}\left(x^{* b}\right)$, then we define $r_{\ell}^{b}=+\infty$ and $u^{b}(-\infty, e(\ell))=\lim _{m \rightarrow+\infty} u^{b}(-m, e(\ell))$. Notice that by the assumptions on $u^{b}$, it holds that $\bar{p}_{\ell}<+\infty$ in this case. It also holds that $u^{b}(-\infty, e(\ell)) \geq$ $u^{b}\left(x^{* b}\right)$. If, for every $m \in \mathbb{R}, u^{b}(-m, e(\ell))<u^{b}\left(x^{* b}\right)$, then we define $r_{\ell}^{b}=-\infty$ and $u^{b}(+\infty, e(\ell))=\lim _{m \rightarrow-\infty} u^{b}(-m, e(\ell))$. It holds that $u^{b}(+\infty, e(\ell)) \leq u^{b}\left(x^{* b}\right)$. Otherwise, let $r_{\ell}^{b} \in \mathbb{R}$ be such that $u^{b}\left(-r_{\ell}^{b}, e(\ell)\right)=u^{b}\left(x^{* b}\right)$. We define $r_{\ell}^{B}=\max _{b \in \beta(\bar{Y} \ell} r_{\ell}^{b}$. 
Consider some $\ell \in L$ and some $s \in \sigma\left(\bar{Y}^{\ell}\right)$. If, for every $m \in \mathbb{R}, u^{s}(m,-e(\ell))>$ $u^{s}\left(x^{* s}\right)$, then we define $r_{\ell}^{s}=-\infty$ and $u^{s}(-\infty,-e(\ell))=\lim _{m \rightarrow-\infty} u^{s}(m,-e(\ell))$. Notice that by the assumptions on $u^{s}$, it holds that $\underline{p}_{\ell}>-\infty$ in this case. It also holds that $u^{s}(-\infty,-e(\ell)) \geq u^{s}\left(x^{* s}\right)$. If, for every $m \in \mathbb{R}, u^{s}(m,-e(\ell))<u^{s}\left(x^{* s}\right)$, then we define $r_{\ell}^{s}=$ $+\infty$ and $u^{s}(+\infty,-e(\ell))=\lim _{m \rightarrow+\infty} u^{s}(m,-e(\ell))$. It holds that $u^{s}(+\infty,-e(\ell)) \leq u^{s}\left(x^{* s}\right)$. Otherwise, let $r_{\ell}^{s} \in \mathbb{R}$ be such that $u^{s}\left(r_{\ell}^{s},-e(\ell)\right)=u^{s}\left(x^{* s}\right)$. We define $r_{\ell}^{S}=\min _{s \in \sigma\left(\bar{Y}^{\ell}\right)} r_{\ell}^{s}$.

It holds by definition that $r_{\ell}^{b}=p_{\ell}^{*}=r_{\ell}^{s}$ for every $(b, s, \ell, m) \in A$.

Step 3. For every $\ell \in \lambda(A)$ at least one of the following three cases holds:

1. $p_{\ell}^{*}=\underline{p}_{\ell}$ and $r_{\ell}^{S} \leq \underline{p}_{\ell}=r_{\ell}^{B}$,

2. $\underline{p}_{\ell}<p_{\ell}^{*}<\bar{p}_{\ell}$ and $r_{\ell}^{S}=p_{\ell}^{*}=r_{\ell}^{B}$,

3. $p_{\ell}^{*}=\bar{p}_{\ell}$ and $r_{\ell}^{S}=\bar{p}_{\ell} \leq r_{\ell}^{B}$.

It follows from the definitions in Step 2 that, for every $(b, s, \ell, m) \in A, r_{\ell}^{b}=p_{\ell}^{*}=r_{\ell}^{s}$, so it follows that $r_{\ell}^{S} \leq p_{\ell}^{*} \leq r_{\ell}^{B}$.

Consider some $\ell \in L$. It clearly holds that a. $p_{\ell}^{*}=\underline{p}_{\ell}$, or b. $\underline{p}_{\ell}<p_{\ell}^{*}<\bar{p}_{\ell}$, or c. $p_{\ell}^{*}=\bar{p}_{\ell}$. Each of the Cases a, b, and $\mathrm{c}$ is considered in turn and shown to lead to at least one of the Cases 1, 2, and 3 of Step 3.

Case a. $p_{\ell}^{*}=p_{\ell}$.

If $r_{\ell}^{B}=\underline{p}_{\ell}$, then Case 1 of Step 3 holds and we are done, so assume $r_{\ell}^{B}>\underline{p}_{\ell}$. We argue that $r_{\ell}^{S}=\underline{p}_{\ell}$. Suppose not, then $r_{\ell}^{S} \leq p_{\ell}^{*}=\underline{p}_{\ell}$ implies $r_{\ell}^{S}<\underline{p}_{\ell}$. Let $b \in \beta\left(\bar{Y}^{\ell}\right)$ be such that $r_{\ell}^{b}=r_{\ell}^{B}$, let $s \in \sigma\left(\bar{Y}^{\ell}\right)$ be such that $r_{\ell}^{s}=r_{\ell}^{S}$, and consider the contract $z=\left(b, s, \ell, \underline{p}_{\ell}\right)$. Since the set of traders in commodity $\ell$ has a product structure, it holds that $z \in \bar{Y}$. The utilities corresponding to $z$ are equal to

$$
\begin{aligned}
& U^{b}(\{z\})=u^{b}\left(-\underline{p}_{\ell}, e(\ell)\right)>u^{b}\left(-r_{\ell}^{b}, e(\ell)\right) \geq u^{b}\left(x^{* b}\right), \\
& U^{s}(\{z\})=u^{s}\left(\underline{p}_{\ell},-e(\ell)\right)>u^{s}\left(r_{\ell}^{s},-e(\ell)\right) \geq u^{s}\left(x^{* s}\right),
\end{aligned}
$$

so Condition 2 of Definition 3.1 is violated and the outcome $A$ is not stable, a contradiction. Consequently, it holds that $r_{\ell}^{S}=\underline{p}_{\ell}$.

We argue next that $\underline{p}_{\ell}=\bar{p}_{\ell}$. Suppose not, then it holds that $\underline{p}_{\ell}<\bar{p}_{\ell}$. Let $b \in \beta\left(\bar{Y}^{\ell}\right)$ be such that $r_{\ell}^{b}=r_{\ell}^{B}$, let $s \in \sigma\left(\bar{Y}^{\ell}\right)$ be such that $r_{\ell}^{s}=r_{\ell}^{S}$, and let $m \in \mathbb{R}$ be such that $\underline{p}_{\ell}<m<\min \left\{\bar{p}_{\ell}, r_{\ell}^{B}\right\}$. The contract $z=(b, s, \ell, m)$ belongs to $\bar{Y}$ since the set of traders in commodity $\ell$ has a product structure and $\underline{p}_{\ell}<m<\bar{p}_{\ell}$. The utilities corresponding to $z$ are equal to

$$
\begin{aligned}
& U^{b}(\{z\})=u^{b}(-m, e(\ell))>u^{b}\left(-r_{\ell}^{b}, e(\ell)\right) \geq u^{b}\left(x^{* b}\right), \\
& U^{s}(\{z\})=u^{s}(m,-e(\ell))>u^{s}\left(\underline{p}_{\ell},-e(\ell)\right)=u^{s}\left(r_{\ell}^{s},-e(\ell)\right) \geq u^{s}\left(x^{* s}\right) .
\end{aligned}
$$


Condition 2 of Definition 3.1 is violated and the outcome $A$ is not stable, a contradiction. Consequently, it holds that $\underline{p}_{\ell}=\bar{p}_{\ell}$. Now Case 3 of Step 3 holds and we are done.

Case b. $\underline{p}_{\ell}<p_{\ell}^{*}<\bar{p}_{\ell}$.

Suppose that Case 2 of Step 3 does not hold. We end up in Subcase (b.i). $r_{\ell}^{S}<p_{\ell}^{*} \leq r_{\ell}^{B}$ or Subcase (b.ii). $r_{\ell}^{S} \leq p_{\ell}^{*}<r_{\ell}^{B}$.

In Subcase (b.i), let $b \in \beta\left(\bar{Y}^{\ell}\right)$ be such that $r_{\ell}^{b}=r_{\ell}^{B}$, let $s \in \sigma\left(\bar{Y}^{\ell}\right)$ be such that $r_{\ell}^{s}=r_{\ell}^{S}$, and let $m \in \mathbb{R}$ be such that $\max \left\{p_{\ell}, r_{\ell}^{S}\right\}<m<p_{\ell}^{*}$. The contract $z=(b, s, \ell, m)$ belongs to $\bar{Y}$ since the set of traders in commodity $\ell$ has a product structure and $p_{\ell}<m<\bar{p}_{\ell}$. The utilities corresponding to $z$ are equal to

$$
\begin{aligned}
& U^{b}(\{z\})=u^{b}(-m, e(\ell))>u^{b}\left(-p_{\ell}^{*}, e(\ell)\right) \geq u^{b}\left(-r_{\ell}^{b}, e(\ell)\right) \geq u^{b}\left(x^{* b}\right), \\
& U^{s}(\{z\})=u^{s}(m,-e(\ell))>u^{s}\left(r_{\ell}^{s},-e(\ell)\right) \geq u^{s}\left(x^{* s}\right),
\end{aligned}
$$

so Condition 2 of Definition 3.1 is violated and the outcome $A$ is not stable, a contradiction.

In Subcase (b.ii), let $b \in \beta\left(\bar{Y}^{\ell}\right)$ be such that $r_{\ell}^{b}=r_{\ell}^{B}$, let $s \in \sigma\left(\bar{Y}^{\ell}\right)$ be such that $r_{\ell}^{s}=r_{\ell}^{S}$, and let $m \in \mathbb{R}$ be such that $p_{\ell}^{*}<m<\min \left\{\bar{p}_{\ell}, r_{\ell}^{B}\right\}$. The contract $z=(b, s, \ell, m)$ belongs to $\bar{Y}$ since the set of traders in commodity $\ell$ has a product structure and $\underline{p}_{\ell}<m<\bar{p}_{\ell}$. The utilities corresponding to $z$ are equal to

$$
\begin{aligned}
& U^{b}(\{z\})=u^{b}(-m, e(\ell))>u^{b}\left(-r_{\ell}^{b}, e(\ell)\right) \geq u^{b}\left(x^{* b}\right), \\
& U^{s}(\{z\})=u^{s}(m,-e(\ell))>u^{s}\left(p_{\ell}^{*},-e(\ell)\right) \geq u^{s}\left(r_{\ell}^{s},-e(\ell)\right) \geq u^{s}\left(x^{* s}\right),
\end{aligned}
$$

so Condition 2 of Definition 3.1 is violated and the outcome $A$ is not stable, a contradiction.

Since both Subcases (b.i) and (b.ii) lead to a contradiction, our supposition is false, so Case 2 of Step 3 holds and we are done.

Case c. $p_{\ell}^{*}=\bar{p}_{\ell}$.

By an argument completely symmetric to the one of Case a, Case 1 or Case 3 of Step 3 can be shown to hold.

Step 4. For every $\ell \in L \backslash \lambda(A)$, at least one of the following three cases holds:

1. $r_{\ell}^{B} \leq \underline{p}_{\ell}$,

2. $\underline{p}_{\ell}<r_{\ell}^{B} \leq r_{\ell}^{S}<\bar{p}_{\ell}$,

3. $r_{\ell}^{S} \geq \bar{p}_{\ell}$.

Suppose not. Then there is $\ell \in L \backslash \lambda(A)$ such that

$$
r_{\ell}^{B}>\underline{p}_{\ell}, r_{\ell}^{S}<\bar{p}_{\ell}, \text { and } r_{\ell}^{B}>r_{\ell}^{S}
$$


Take $b \in \beta\left(\bar{Y}^{\ell}\right)$ with $r_{\ell}^{b}=r_{\ell}^{B}$ and $s \in \sigma\left(\bar{Y}^{\ell}\right)$ with $r_{\ell}^{s}=r_{\ell}^{S}$. Since $r_{\ell}^{B} \neq-\infty$ and $r_{\ell}^{S} \neq+\infty$, it holds that $u^{b}\left(-r_{\ell}^{b}, e(\ell)\right) \geq u^{b}\left(x^{* b}\right)$ and $u^{s}\left(r_{\ell}^{s},-e(\ell)\right) \geq u^{s}\left(x^{* s}\right)$. Let $m \in \mathbb{R}$ be such that $\underline{p}_{\ell} \leq m \leq \bar{p}_{\ell}$ and $r_{\ell}^{S}<m<r_{\ell}^{B}$. Since $\underline{p}_{\ell} \leq m \leq \bar{p}_{\ell}$ and the set of traders in commodity $\ell$ has a product structure, it holds that $z=(b, s, m, \ell) \in \bar{Y}$. The utilities corresponding to $z$ are equal to

$$
\begin{aligned}
& U^{b}(\{z\})=u^{b}(-m, e(\ell))>u^{b}\left(-r_{\ell}^{b}, e(\ell)\right) \geq u^{b}\left(x^{* b}\right), \\
& U^{s}(\{z\})=u^{s}(m,-e(\ell))>u^{s}\left(r_{\ell}^{s},-e(\ell)\right) \geq u^{s}\left(x^{* s}\right),
\end{aligned}
$$

so Condition 2 of Definition 3.1 is violated and the outcome $A$ is not stable, a contradiction. The result as stated in Step 4 follows.

Step 5. Definition of prices for non-traded commodities.

Choose any $\ell \in L \backslash \lambda(A)$. Using Step 4, exactly one of the following three cases holds.

Case 1. $r_{\ell}^{B} \leq \underline{p}_{\ell}$. If $\underline{p}_{\ell}>-\infty$, then define $p_{\ell}^{*}=\underline{p}_{\ell}$. Consider the case $\underline{p}_{\ell}=-\infty$. Since $\underline{p}_{\ell}=-\infty$, it holds that $r_{\ell}^{S}>-\infty$. Define $p_{\ell}^{*}=\min \left\{\bar{p}_{\ell}, r_{\ell}^{S}, 0\right\}$.

Case 2. $\underline{p}_{\ell}<r_{\ell}^{B} \leq r_{\ell}^{S}<\bar{p}_{\ell}$. Define $p_{\ell}^{*}=\left(r_{\ell}^{B}+r_{\ell}^{S}\right) / 2$.

Case 3. $\underline{p}_{\ell}<r_{\ell}^{B}$ and $\bar{p}_{\ell} \leq r_{\ell}^{S}$. If $\bar{p}_{\ell}<+\infty$, then define $p_{\ell}^{*}=\bar{p}_{\ell}$. If $\bar{p}_{\ell}=+\infty$, then define $p_{\ell}^{*}=r_{\ell}^{B}$.

Step 6. Definition of rationing constraints.

For every buyer, we introduce rationing constraints for those commodities that he does not trade but would like to trade and which have a price equal to the price ceiling,

$$
Q^{*}=\left\{(b, \ell) \in B \times L \mid \ell \in L^{b} \backslash \lambda\left(A^{b}\right) \text { and } r_{\ell}^{b}>p_{\ell}^{*}=\bar{p}_{\ell}\right\} .
$$

For every seller, we introduce rationing constraints for those commodities that he does not trade but would like to trade and which have a price equal to the price floor,

$$
R^{*}=\left\{(s, \ell) \in S \times L \mid \ell \in L^{s} \backslash \lambda\left(A^{s}\right) \text { and } r_{\ell}^{s}<p_{\ell}^{*}=\underline{p}_{\ell}\right\} .
$$

Step 7. $\left(p^{*}, Q^{*}, R^{*}, x^{*}\right)$ satisfies Condition 1 of Definition 5.1.

Consider some $b \in B$. Suppose $x^{* b} \notin \delta^{b}\left(p^{*}, Q^{* b}\right)$. Take some $x^{b} \in \delta^{b}\left(p^{*}, Q^{* b}\right)$. It follows that $x^{b} \neq 0$ since otherwise $A^{b} \notin C^{b}(A)$, thereby violating Condition 1 of Definition 3.1. Let $\ell \in L^{b}$ be such that $x_{\ell}^{b}=1$. It follows that $\ell \notin Q^{* b}$. By (7.1) it must be that $\ell \in \lambda\left(A^{b}\right)$ or $r_{\ell}^{b} \leq p_{\ell}^{*}$ or $p_{\ell}^{*}<\bar{p}_{\ell}$. The first two cases imply $u^{b}\left(x^{b}\right)=u^{b}\left(x^{* b}\right)$ and $u^{b}\left(x^{b}\right) \leq u^{b}\left(x^{* b}\right)$, respectively, leading to a contradiction. For the last case we distinguish two subcases: (i) $\ell \in L^{b} \backslash \lambda(A)$ and (ii) $\ell \in \lambda(A)$. 
For subcase (i), using $p_{\ell}^{*}<\bar{p}_{\ell}$, it follows from the definition in Step 5 that $r_{\ell}^{B} \leq p_{\ell}^{*}$. At the same time, $u^{b}\left(x^{b}\right)>u^{b}\left(x^{* b}\right)$ implies $p_{\ell}^{*}<r_{\ell}^{b} \leq r_{\ell}^{B}$, so we have obtained a contradiction.

For subcase (ii), since $u^{b}\left(x^{b}\right)>u^{b}\left(x^{* b}\right)$, it holds that $p_{\ell}^{*}<r_{\ell}^{b} \leq r_{\ell}^{B}$, so Cases 1 and 2 of Step 3 do not hold. Since $p_{\ell}^{*}<\bar{p}_{\ell}$, Case 3 of Step 3 is violated as well, so we have obtained a contradiction. Consequently, it holds that $x^{* b} \in \delta^{b}\left(p^{*}, Q^{* b}\right)$.

Step 8. $\left(p^{*}, Q^{*}, R^{*}, x^{*}\right)$ satisfies Condition 2 of Definition 5.1.

Consider some $s \in S$. Suppose $x^{* s} \notin \delta^{s}\left(p^{*}, R^{* s}\right)$. Take some $x^{s} \in \delta^{s}\left(p^{*}, R^{* s}\right)$. It follows that $x^{s} \neq 0$ since otherwise $A^{s} \notin C^{s}(A)$, thereby violating Condition 1 of Definition 3.1. Let $\ell \in L^{s}$ be such that $x_{\ell}^{s}=1$. It follows that $\ell \notin R^{* s}$. By (7.2) it must be that $\ell \in \lambda\left(A^{s}\right)$ or $r_{\ell}^{s} \geq p_{\ell}^{*}$ or $p_{\ell}^{*}>\underline{p}_{\ell}$. The first two cases imply $u^{s}\left(x^{s}\right)=u^{s}\left(x^{* s}\right)$ and $u^{s}\left(x^{s}\right) \leq u^{s}\left(x^{* s}\right)$, respectively, leading to a contradiction. For the last case we distinguish two subcases: (i) $\ell \in L^{s} \backslash \lambda(A)$ and (ii) $\ell \in \lambda(A)$.

For subcase (i), using $p_{\ell}^{*}>\underline{p}_{\ell}$, it follows from the definition in Step 5 that $r_{\ell}^{S} \geq p_{\ell}^{*}$. At the same time, $u^{s}\left(x^{s}\right)>u^{s}\left(x^{* s}\right)$ implies $p_{\ell}^{*}>r_{\ell}^{s} \geq r_{\ell}^{S}$, so we have obtained a contradiction.

For subcase (ii), since $u^{s}\left(x^{s}\right)>u^{s}\left(x^{* s}\right)$, it holds that $p_{\ell}^{*}>r_{\ell}^{s} \geq r_{\ell}^{S}$, so Cases 2 and 3 of Step 3 do not hold. Since $p_{\ell}^{*}>\underline{p}_{\ell}$, Case 1 of Step 3 is violated as well, so we have obtained a contradiction. Consequently, it holds that $x^{* s} \in \delta^{s}\left(p^{*}, R^{* s}\right)$.

Step 9. $\left(p^{*}, Q^{*}, R^{*}, x^{*}\right)$ satisfies Conditions $3,4,5$, and 6 of Definition 5.1.

It follows from (3.1) that

$$
\sum_{i \in I} x^{* i}=\sum_{i \in I} \xi^{i}(A)=0
$$

and therefore Condition 3 of Definition 5.1 is satisfied.

The definition of prices for traded commodities in Step 2 implies that for every $\ell \in \lambda(A)$, $\underline{p}_{\ell} \leq p_{\ell}^{*} \leq \bar{p}_{\ell}$. The definition of prices for non-traded commodities in Step 5 implies that for every $\ell \in L \backslash \lambda(A), \underline{p}_{\ell} \leq p_{\ell}^{*} \leq \bar{p}_{\ell}$. Therefore, Condition 4 of Definition 5.1 is satisfied.

It follows from (7.1) that, for every $\ell \in L$, if $p_{\ell}^{*}<\bar{p}_{\ell}$, then $Q_{\ell}^{*}=\emptyset$, and therefore Condition 5 of Definition 5.1 is satisfied. It follows from (7.2) that, for every $\ell \in L$, if $p_{\ell}^{*}>\underline{p}_{\ell}$, then $R_{\ell}^{*}=\emptyset$, and therefore Condition 6 of Definition 5.1 is satisfied.

Step 10. $\left(p^{*}, Q^{*}, R^{*}, x^{*}\right)$ satisfies Condition 7 of Definition 5.1.

Suppose there is $\ell \in L$ such that $Q_{\ell}^{*} \neq \emptyset$ and $R_{\ell}^{*} \neq \emptyset$. From (7.1) and (7.2) it follows that there is $b \in B$ and $s \in S$ such that $\underline{p}_{\ell}=p_{\ell}^{*}=\bar{p}_{\ell}$ and $r_{\ell}^{s}<p_{\ell}^{*}<r_{\ell}^{b}$, so in particular 
$r_{\ell}^{S}<p_{\ell}^{*}<r_{\ell}^{B}$. If $\ell \in \lambda(A)$, then none of the cases in Step 3 holds, a contradiction. If $\ell \in L \backslash \lambda(A)$, then none of the cases in Step 4 holds, a contradiction. Consequently, for every $\ell \in L, Q_{\ell}^{*}=\emptyset$ or $R_{\ell}^{*}=\emptyset$, and Condition 7 of Definition 5.1 is satisfied.

We conclude that $\left(p^{*}, Q^{*}, R^{*}, x^{*}\right)$ is a Drèze equilibrium and therefore $x^{*} \in X^{\mathrm{D}}$. 


\section{References}

Agell, J., And K.E. Lommerud (1997), "Minimum Wages and the Incentives for Skill Formation," Journal of Public Economics, 64, 25-40.

Andersson, T., And L.G. Svensson (2014), "Non-manipulable House Allocation with Rent Control," Econometrica, 82, 507-539.

Aumann, R.J. (1964), "Markets with a Continuum of Traders," Econometrica, 32, 39-50.

BÉnAssy, J.-P. (1975), "Neo-Keynesian Disequilibrium Theory in a Monetary Economy," Review of Economic Studies, 42, 503-523.

Bulow, J., And P. Klemperer (2012), "Regulated Prices, Rent Seeking, and Consumer Surplus," Journal of Political Economy, 120, 160-186.

Citanna, A., H. Crès, J. Drèze, P.J.J. Herings, And A. Villanacci (2001), "Continua of Underemployment Equilibria Reflecting Coordination Failures, Also at Walrasian Prices," Journal of Mathematical Economics, 36, 169-200.

Corominas-Bosch, M. (2004), "Bargaining in a Network of Buyers and Sellers," Journal of Economic Theory, 115, 35-77.

Cox, C.C. (1980), "The Enforcement of Public Price Controls," Journal of Political Economy, 88, 887916.

Debreu, G., And H. SCArf (1963), "A Limit Theorem on the Core of an Economy," International Economic Review, 4, 235-246.

Demange, G., And D. Gale (1985), "The Strategy Structure of Two-sided Matching Markets," Econometrica, 53, 873-888.

Demange, G., D. Gale, and M. Sotomayor (1986), "Multi-Item Auctions," Journal of Political Economy, 94, 863-872.

DrÈze, J.H. (1975), "Existence of an Exchange Equilibrium under Price Rigidities," International Economic Review, 16, 301-320.

DrÈze, J.H., AND C. Gollier (1993), "Risk Sharing on the Labour Market and Second-best Wage Rigidities," European Economic Review, 37, 1457-1482.

Edgeworth, F.Y. (1881), Mathematical Psychics, Kegan Paul, London.

Feldstein, M. (1973), "The Economics of the New Unemployment," Public Interest, 33, 3-42.

Gale, D., And L.S. Shapley (1962), "College Admissions and the Stability of Marriage," American Mathematical Monthly, 69, 9-15.

Gul, F., And E. Stacchetti (1999), "Walrasian Equilibrium with Gross Substitutes," Journal of Economic Theory, 87, 95-124.

Hatfield, J.W., And S.D. Kominers (2012), "Matching in Networks with Bilateral Contracts," American Economic Journal: Microeconomics, 4, 176-208.

Hatfield, J.W., S.D. Kominers, A. Nichifor, M. Ostrovsky, and A. Westkamp (2013), "Stability and Competitive Equilibrium in Trading Networks," Journal of Political Economy, 121, 966-1005. 
Hatfield, J.W., And P. Milgrom (2005), "Matching with Contracts," American Economic Review, 95, 915-935.

Hatfield, J.W., C.R. Plott, and T. Tanaka (2012A), "Price Controls, Non-price Quality Competition, and the Nonexistence of Competitive Equilibrium," Working Paper, 1-54.

Hatfield, J.W., C.R. Plott, and T. Tanaka (2012B), "Understanding Price Controls and Nonprice Competition with Matching Theory," American Economic Review, Papers 83 Proceedings, 102, 371375 .

Herings, P.J.J. (1996), Static and Dynamic Aspects of General Disequilibrium Theory, Theory and Decision Library, Series C: Game Theory, Mathematical Programming and Operations Research, Kluwer Academic Publishers, Norwell, Massachusetts.

Herings, P.J.J. (2014), "General Equilibrium and the New Neoclassical Synthesis," Economic Theory, $57,437-477$.

Herings, P.J.J., And A. Konovalov (2009), "Constrained Suboptimality when Prices Are Non-competitive," Journal of Mathematical Economics, 45, 43-58.

Herings, P.J.J., And H.M. Polemarchakis (2005), "Pareto Improving Price Regulation when the Asset Market Is Incomplete," Economic Theory, 25, 135-154.

Kelso, A.S., Jr., And V.P. Crawford (1982), "Job Matching, Coalition Formation, and Gross Substitutes," Econometrica, 50, 1483-1504.

Leffler, K.B. (1982), “Ambiguous Changes in Product Quality," American Economic Review, 72, 956967.

Levy, S. (1991), "The Short-Term Macroeconomic Effects of Price Controls," Journal of Policy Modeling, 13, 157-184.

Maskin, E.S. (2000), "Auctions, Development, and Privatization: Efficient Auctions with Liquidityconstrained Buyers," European Economic Review, 44, 667-681.

Nakamura, E., And J. Steinsson (2008), "Five Facts about Prices: A Reevaluation of Menu Cost Models," Quarterly Journal of Economics, 123, 1415-1464.

NAKamura, E., And J. Steinsson (2010), "Monetary Non-neutrality in a Multisector Menu Cost Model," Quarterly Journal of Economics, 125, 961-1013.

Nguyen, T.T., And J. Whalley (1990), "General Equilibrium Analysis of Price Controls: A Computational Approach," International Economic Review, 31, 667-684.

Perloff, J.M. (2012), Microeconomics, Sixth Edition, Pearson Education, Harlow.

Polemarchakis, H.M. (1979), "Incomplete Markets, Price Regulation, and Welfare," American Economic Review, 69, 662-669.

Rотн, A.E. (1991), "A Natural Experiment in the Organization of Entry-level Labor Markets: Regional Markets for New Physians and Surgeons in the United Kingdom," American Economic Review, 81, 415-440.

Roth, A.E., And M.A.O. Sotomayor (1990), Two-sided Matching, A Study in Game-theoretic Modeling and Analysis, Econometric Society Monographs No. 18, Cambridge University Press, Cambridge. 
Shapley, L.S., And M. Shubik (1971), "The Assignment Game I: The Core," International Journal of Game Theory, 1, 111-130.

Sun, N., AND Z. YAng (2006), "Equilibria and Indivisibilities: Gross Substitutes and Complements," Econometrica, 74, 1385-1402.

Talman, A.J.J., AND Z. YAng (2008), "A Dynamic Auction for Differentiated Items under Price Rigidities," Economics Letters, 99, 278-281.

Woodford, M. (2003), Interest \& $\mathcal{3}$ Prices, Foundations of a Theory of Monetary Policy, Princeton University Press, Princeton, New Jersey.

YAng, Z., And D. Zhang (2013), "On a Fundamental Property of Talman-Yang's Auction under Price Control," Discussion Paper No. 13/06, The University of York, 1-29.

Younès, Y. (1975), "On the Role of Money in the Process of Exchange and the Existence of a NonWalrasian Equilibrium," Review of Economic Studies, 42, 489-501. 